\title{
The Spectral Flow for Dirac Operators on Compact Planar Domains with Local Boundary Conditions
}

\author{
Marina Prokhorova \\ Ural Federal University, Ekaterinburg, Russia. E-mail: pmf@imm.uran.ru
}

Received: 28 May 2012 / Accepted: 16 October 2012

Published online: 29 March 2013 - @ Springer-Verlag Berlin Heidelberg 2013

\begin{abstract}
Let $D_{t}, 0 \leqslant t \leqslant 1$ be a 1-parameter family of Dirac type operators on a two-dimensional disk with $m-1$ holes. Suppose that all operators $D_{t}$ have the same symbol, and that $D_{1}$ is conjugate to $D_{0}$ by a scalar gauge transformation. Suppose that all operators $D_{t}$ are considered with the same elliptic local boundary condition. Our main result is a computation of the spectral flow for such a family of operators. The answer is obtained up to multiplication by an integer constant depending only on the number of holes in the disk. This constant is calculated explicitly for the case of the annulus $(m=2)$.
\end{abstract}

\section{Contents}

1. Introduction . . . . . . . . . . . . . . . . . 386

I The Spectral Flow for Dirac Operators . . . . . . . . . . . . . . . . . . . . 388

2. The Spectral Flow . . . . . . . . . . . . . . . . . . . . . 388

3. Dirac Operators: the Simplest Case . . . . . . . . . . . . . . . . . . . . . 389

4. 2N-dimensional Dirac operators . . . . . . . . . . . . . . . . . . . . . . . . . . 392

5. Dirac Type Operators . . . . . . . . . . . . . . . . . . . . 392

6. The Case of One Hole . . . . . . . . . . . . . . . . . . . . . 395

7. The Case of Several Holes . . . . . . . . . . . . . . . . . . . . . . . . . . . . . . . 396

8. General Case: First Order Elliptic Operators . . . . . . . . . . . . . . . . 397

9. The Spectral Flow for $\mathrm{N}=2$ in Terms of Condensed Matter Physics . . . . 398

II Proof of Theorem $3 \ldots \ldots$. . . . . . . . . . . . . . . . . . . . . . . . . . 402

10. Two Technical Lemmas . . . . . . . . . . . . . . . . . . . . . . . . . . . . . . . . . . . . . . . . . .

11. Basic Properties of the Spectral Flow . . . . . . . . . . . . . . . 405

12. Independence of the Choice of the Family $\left(Q_{t}\right) \ldots \ldots$. . . . . . . . . . . . . . . . . . . 407

13. Independence of the Choice of Operator D . . . . . . . . . . . . . 407

14. Independence of the Metric and Invariance Under a Change of Variables . 409

15. Boundary Conditions . . . . . . . . . . . . . . . . . . 410 
16. Gauge Transformations . . . . . . . . . . . . . . . 411

17. Bilinearity ..................... 411

18. Invariance under the Action of Symmetric Group . . . . . . . . . . . 413

Acknowledgements . . . . . . . . . . . . . . . . . . . 413

Reference . . . . . . . . . . . . . . . . . . . . . . . . 414

\section{Introduction}

This paper deals with Dirac type operators on compact planar domains. We consider such operators with self-adjoint locally elliptic local boundary conditions. ${ }^{1}$ The paper is focused not on individual operators, but on paths in the space of such operators. We consider only paths connecting two operators conjugate by a scalar gauge transformation (so, they are loops up to a scalar gauge transformation). Such paths have a well known invariant, the spectral flow, which counts with signs the number of eigenvalues passing through zero from the start of the path to its end (the eigenvalues passing from negative values to positive ones are counted with the plus sign, and eigenvalues passing in the other direction are counted with the minus sign). The paper is devoted to the problem of computation of the spectral flow in the situation when all operators along a given path have the same symbol and the same boundary condition.

Because these results are potentially useful for the physics of condensed matter, the author has attempted to avoid advanced mathematical terminology and to explain the results and the ideas behind their proofs in a way accessible to non-mathematicians. For the same reason, the case of classical Dirac operators (Theorems 1 and 2) is treated before dealing with the more general case of Dirac type operators on domains equipped with an arbitrary metric. Note that physicists sometimes use more general boundary conditions than the ones considered in this paper. For example, the so-called armchair boundary conditions for graphene are of the type considered in this paper, but the zigzag boundary conditions for graphene are not. While it is not completely obvious, the boundary conditions considered in this paper are just other forms of the locally elliptic boundary conditions usually used in physics, as explained in Sect. 9. Besides, we explain in Sect. 9 how the spectral flow can be computed in terms of the general boundary problem for two-dimensional spinors considered by Akhmerov and Beenakker in [1]. As illustration we show that the spectral flow vanishes if the boundary condition does not break time reversal symmetry.

We start with the following situation. Let $\mathrm{X}$ be a compact planar domain bounded by $m$ smooth curves (topologically it is a disk with $m-1$ holes). Our operators act on spinor-valued functions, which we identify with column vectors of two complex-valued functions:

$$
u=\left(\begin{array}{l}
u^{+} \\
u^{-}
\end{array}\right), \quad u^{ \pm}: X \rightarrow \mathbb{C} .
$$

A Dirac operator acting on spinor functions has the form

$$
D=\mathbb{D}+\left(\begin{array}{cc}
0 & \bar{q}(x) \\
q(x) & 0
\end{array}\right), \quad \mathbb{D}=-i\left(\begin{array}{cc}
0 & \partial_{1}-i \partial_{2} \\
\partial_{1}+i \partial_{2} & 0
\end{array}\right),
$$

where $q$ is a smooth function from $X$ to $\mathbb{C}, x=\left(x^{1}, x^{2}\right) \in X, \partial_{i}=\partial / \partial x^{i}$. Our focus is on 1-parameter families $D_{t}$ of such operators parameterized by $t \in[0,1]$. In such a

\footnotetext{
${ }^{1}$ In particular, boundary conditions defined by general pseudo-differential operators are not allowed.
} 
family the first term $\mathbb{D}$ involving derivatives is always the same, but in the second term the function $q$ is allowed to change continuously with $t$, i.e. $q=q_{t}$, where $t \in[0,1]$. In agreement with the above, we assume that $D_{1}=\mu D_{0} \mu^{-1}$ for some smooth scalar gauge transformation $\mu: \mathrm{X} \rightarrow \mathrm{U}(1)$.

All operators $D_{t}$ are considered with the same boundary condition of the form $i\left(n_{1}+\right.$ $\left.\mathrm{in}_{2}\right) \mathrm{u}^{+}=\mathrm{B}(\mathrm{x}) \mathrm{u}^{-}$, where $\mathrm{n}=\left(\mathrm{n}_{1}, \mathrm{n}_{2}\right)$ is the outward conormal to the boundary, and $B$ is a real-valued smooth function without zeros, defined on the boundary of $X$. Our first main result, Theorem 1 , asserts that the spectral flow of such a family of operators is equal to $c_{m} \sum_{j=1}^{m} b_{j} \mu_{j}$, where $c_{m}$ is an integer constant depending on $m$ only, $\mu_{j}$ is the degree of the restriction of $\mu$ to the $j^{\text {th }}$ connected boundary component, $b_{j}=1$ if $B$ is negative on the $j^{\text {th }}$ boundary component, and equal to 0 otherwise.

After considering this most special and very important situation, we turn our attention to the situation of Dirac operators acting on $\mathrm{N}$-dimensional spinor functions

$$
u=\left(\begin{array}{l}
u^{+} \\
u^{-}
\end{array}\right), u^{ \pm}: X \rightarrow \mathbb{C}^{N},
$$

where, as before, $\mathrm{X}$ is a compact planar domain bounded by $\mathrm{m}$ smooth curves. A Dirac operator acting on $\mathrm{N}$-dimensional spinor functions has the form

$$
\mathrm{D}=\mathbb{D}+\mathrm{Q}(x), \mathbb{D}=-\mathrm{i}\left(\sigma_{1} \partial_{1}+\sigma_{2} \partial_{2}\right),
$$

where

$$
\sigma_{1}=\left(\begin{array}{cc}
0 & \mathrm{I}_{\mathrm{N}} \\
\mathrm{I}_{\mathrm{N}} & 0
\end{array}\right), \sigma_{2}=\left(\begin{array}{cc}
0 & -i \mathrm{I}_{\mathrm{N}} \\
i \mathrm{I}_{\mathrm{N}} & 0
\end{array}\right),
$$

$I_{N}$ is the $N \times N$ unit matrix, and $Q(x)$ is a complex self-adjoint $2 N \times 2 N$ matrix smoothly dependent on $x \in X$. Again, our focus is on 1-parameter families $D_{t}$ of such operators parameterized by $t \in[0,1]$. In such a family the first term $\mathbb{D}$ involving derivatives is always the same, but in the second term the matrix $Q$ is allowed to change continuously with $t$, i. e. $Q=Q_{t}$, where $t \in[0,1]$. We assume that $D_{1}=\mu D_{0} \mu^{-1}$ for some smooth scalar gauge transformation $\mu: X \rightarrow U(1)$, where $\mathrm{U}(1)$ is identified with the subgroup of $\mathrm{U}(2 \mathrm{~N})$ consisting of diagonal matrices with equal diagonal elements.

All operators $D_{t}$ are considered with the same boundary condition $i\left(n_{1}+i n_{2}\right) u^{+}=$ $\mathrm{B}(x) \mathrm{u}^{-}$, where $\mathrm{B}$ is a smooth map from the boundary to the space of complex self-adjoint invertible $\mathrm{N} \times \mathrm{N}$ matrices. Note that a local boundary condition is locally elliptic if and only if it can be written in such a form with $B(x)$ invertible at any $x$; this boundary condition is self-adjoint if and only if $B(x)$ is self-adjoint at any $x$.

Our second main result, Theorem 2, asserts that the spectral flow of such a family of operators is equal to $c_{m} \sum_{j=1}^{m} b_{j} \mu_{j}$, where $c_{m}$ is the same constant as in Theorem 1 (in particular, $c_{m}$ does not depend on the dimension $\left.N\right), \mu_{j}$ is the degree of the restriction of $\mu$ to the $j^{\text {th }}$ boundary component (this restriction gives us a map from the circle to the circle because $\mu$ is a scalar gauge transformation), and $b_{j}$ is the number of negative eigenvalues of $B(x)$ (counted with multiplicities) on the $j^{\text {th }}$ boundary component.

Theorem 3 extends Theorem 2 to a still more general class of operators. These Dirac type operators involve in their definition an arbitrary (not necessarily flat) metric on $X$, and have principal symbol defined by a Clifford multiplication which does not necessarily agree with this metric. While considering an arbitrary metric is important for some physical applications, considering Clifford multiplication which does not agree with the metric on $\mathrm{X}$ does not seem necessary. Nevertheless, we take care of this more general 
case because the proofs of our results crucially depend on it. Moreover, we cannot prove Theorems 1 and 2 without proving Theorem 3 first.

Notice that scalar gauge transformations $\mu: X \rightarrow U(1)$ leave invariant every local boundary condition, as well as the first term $\mathbb{D}$ of the operator $\mathbb{D}+Q(x): \mu(\mathbb{D}+Q) \mu^{-1}=$ $\mathbb{D}+\mathrm{Q}^{\prime}$ for some function $\mathrm{Q}^{\prime}(x)$. So any operator $\mathrm{D}_{0}$ can be connected with the conjugate operator $D_{1}=\mu D_{0} \mu^{-1}$ by the path $\left(\mathbb{D}+Q_{t}(x)\right)$ with fixed boundary condition. On the contrary, non-scalar gauge transformations $\mu: X \rightarrow U(2 N)$ do not have such properties, so the problem of computing the spectral flow cannot be stated in such a form as described above. If we allow general non-scalar gauge transformations, then we have to allow paths of operators $\left(D_{t}\right)$ and of boundary conditions $\left(B_{t}\right)$ with $B_{t}$ and the symbol of $D_{t}$ being dependent on $t$. Some results about this more general case are outlined in Sect. 8.

In this paper the spectral flow is computed only up to multiplication by an integer constant $c_{m}$ depending only on $m$. For a disk with one hole $(m=2)$ the eigenvectors and hence the spectral flow are calculated explicitly in a special case; this is sufficient to determine $c_{2}$; it turns out that $c_{2}=1$ (see Theorem 4 ). In the case of $m>2$ such explicit computations are hardly possible, but from the very beginning of her work the author conjectured that $\mathrm{c}_{\mathrm{m}}=1$ for all $\mathrm{m}$; a partial motivation for this conjecture is provided in Sect. 7. After the preprint version [15] of this paper was distributed, M. I. Katsnelson and V. E. Nazaikinski posted preprint [11], in which they proved, in particular, this conjecture of the author.

It was pointed out by one of the referees, that the works of T. Yoshida [18], L. Nicolaescu [13], and B. Booss-Bavnbek and K. Furutani [4] allow the results of this paper to be interpreted as an alternative way to compute the Maslov index of corresponding family of the Cauchy data spaces intersected with the trace of the domain at the boundary.

\section{Part I. The Spectral Flow for Dirac Operators.}

\section{The Spectral Flow}

Let $H$ be a complex separable Hilbert space, $\left(A_{t}\right), t \in[0,1]$ be a continuous 1 -parameter family of bounded self-adjoint (or, what is the same, Hermitian) Fredholm operators in $H$. Every $A_{t}$ has real spectrum. The neighbourhood of zero in this spectrum is discrete and changes continuously with the variation of $t$. Hence one can count the net number of eigenvalues of $A_{t}$ passing through zero in the positive direction as $t$ runs from 0 to 1 , that is, the difference between the numbers of eigenvalues (counting multiplicities) crossing zero in positive and negative directions. This net number is called the spectral flow $\operatorname{sf}\left(A_{t}\right)$. A description of this notion can be found in $[2,14]$.

The case when $A_{0}$ or $A_{1}$ has zero eigenvalue requires some agreement on the counting procedure; we use the following convention: take a small $\varepsilon>0$ such that $A_{0}, A_{1}$ have no eigenvalues in the interval $[-\varepsilon, 0)$, and define the spectral flow as the net number of eigenvalues of $A_{t}+\varepsilon I$ which pass through zero.

Let now $\left(A_{t}\right)$ be a 1-parameter family of (not necessarily bounded) self-adjoint Fredholm operators in $\mathrm{H}$. For example, it can be a family of symmetric elliptic differential operators $A_{t}$ acting on sections of a Hermitian bundle $E$ over a closed (that is, compact without boundary) manifold $X$. The definition of the spectral flow can be adjusted to this case, though more careful consideration is needed, particularly due to the presence of various natural topologies on the space of such operators $[5,6,12]$. 
When a manifold has non-empty boundary, we have to consider the family $\left(A_{t}, B_{t}\right)$, where $A_{t}$ is a formally self-adjoint elliptic differential operator, and $B_{t}$ is a "good" (self-adjoint elliptic) boundary condition for $A_{t}$ at any $t$. One can see the notion of self-adjoint elliptic boundary value problem for operators of Dirac type in [8,9], and for general first order elliptic operators in [7].

Such a differential operator $A_{t}$ with boundary condition $B_{t}$ defines an unbounded self-adjoint Fredholm operator on $\mathrm{L}^{2}(X, E)$, which has unbounded discrete real spectrum. Intuitively, the spectrum of $\left(A_{t}, B_{t}\right)$ changes continuously with the variation of $t$, so the definition of the spectral flow works in this case as well $[5,6]$. However, the proof that the definition and the standard properties of the spectral flow are correct is considerably more difficult in this case. The crucial ingredient is the continuity (in $t$ ) of the family $\left(A_{t}, B_{t}\right)$ in the space of unbounded self-adjoint Fredholm operators on $\mathrm{L}^{2}(\mathrm{X}, \mathrm{E})$ with an appropriate metric. This was proved in [7] (see [7], Thm. 7.16). This continuity property allows one to use the theory developed in $[6,12]$ in full force. Our proof of Theorem 3 (see Part II) depends crucially on this theory, and, in particular, on Theorem 7.16 from [7]. The results of this theory needed for the proof of Theorem 3 are isolated in Sect. 11 as properties (P0-P4).

Note that if the spectra of $\left(A_{0}, B_{0}\right)$ and $\left(A_{1}, B_{1}\right)$ are the same (isospectral operators), which is the case in this paper, then there is another way to define the spectral flow of $\left(A_{t}, B_{t}\right)$. The set

$$
\left\{(t, \lambda): \lambda \text { is an eigenvalue of }\left(A_{t}, B_{t}\right)\right\}
$$

can be uniquely represented as the union of the graphs of functions $\lambda_{i}(t)$ such that $\lambda_{i}(t) \leqslant \lambda_{j}(t)$ for $i \leqslant j$. This collection of functions gives us a bijection (one-to-one correspondence) of the spectrum of $\left(A_{0}, B_{0}\right)$ to the spectrum of $\left(A_{1}, B_{1}\right)$. If these two spectra coincide as subsets of $\mathbb{R}$ then this correspondence gives us the shift of the spectrum on the integer number of positions. This number is the spectral flow of $\left(A_{t}, B_{t}\right)$; it is denoted by $\operatorname{sf}\left(A_{t}, B_{t}\right)$. It is worth noting that for the isospectral case one can replace the level $\lambda=0$ by any real number, and the difference between eigenvalues crossing the level in positive and negative directions will be the same [2].

\section{Dirac Operators: the Simplest Case}

Suppose $X$ is a compact planar domain bounded by $m$ smooth curves (topologically it is a disk with $m-1$ holes $)$. We will use the notations $x=\left(x^{1}, x^{2}\right) \in X, \partial_{i}=\partial / \partial x^{i}$.

Let us consider the Dirac operator on $\mathrm{X}$,

$$
\mathbb{D}=-i\left(\begin{array}{cc}
0 & \partial_{1}-i \partial_{2} \\
\partial_{1}+i \partial_{2} & 0
\end{array}\right)
$$

acting on a spinor function $u: X \rightarrow \mathbb{C}^{2}, u=\left(\begin{array}{l}u^{+} \\ u^{-}\end{array}\right)$.

A Dirac operator with non-zero vector potential has the form

$$
\mathrm{D}=\mathbb{D}+\mathrm{Q}(\mathrm{x}), \text { where } \mathrm{Q}(\mathrm{x})=\left(\begin{array}{cc}
0 & \overline{\mathrm{q}}(\mathrm{x}) \\
\mathrm{q}(\mathrm{x}) & 0
\end{array}\right),
$$

$\mathrm{q}$ is a smooth function from $\mathrm{X}$ to $\mathbb{C}$. 
Let $\mu: X \rightarrow U(1)$ be a gauge transformation; we suppose that $\mu(x) \in \mathbb{C},|\mu(x)| \equiv 1$ for $x \in X$. Let us take a Dirac operator $D_{0}=\mathbb{D}+Q_{0}(x)$ and connect it with the conjugate operator

$$
\mathrm{D}_{1}=\mu \mathrm{D}_{0} \mu^{-1}=\mathbb{D}+\mathrm{Q}_{0}+\left(\begin{array}{cc}
0 & i \mu^{-1}\left(\partial_{1} \mu-i \partial_{2} \mu\right) \\
i \mu^{-1}\left(\partial_{1} \mu+i \partial_{2} \mu\right) & 0
\end{array}\right)
$$

by a one-parameter family of Dirac operators

$$
D_{t}=\mathbb{D}+Q_{t}, \quad \text { where } Q_{t}(x)=\left(\begin{array}{cc}
0 & \bar{q}_{t}(x) \\
q_{t}(x) & 0
\end{array}\right),
$$

$q_{t}$ is a smooth function from $X$ to $\mathbb{C}$ continuously depending on $t \in[0,1]$,

$$
q_{1}-q_{0}=i \mu^{-1}\left(\partial_{1} \mu+i \partial_{2} \mu\right) .
$$

A self-adjoint locally elliptic ${ }^{2}$ local boundary condition for $D_{t}$ has the form

$$
\text { in }(x) u^{+}=B(x) u^{-} \quad \text { on } \partial x
$$

where $\mathrm{B}: \partial X \rightarrow \mathbb{R} \backslash\{0\}$ is a smooth function defining the boundary condition, $\mathrm{n}=$ $\left(n_{1}, n_{2}\right)$ is the outward conormal to the boundary $\partial X$ of $X$ at point $x$, and we identify $n$ with the complex number $n_{1}+i n_{2}$ in (3).

Note that $n_{1}, n_{2}$ coincide with the components of the outward normal to $\partial X$ for the case of Euclidean metric considered both here and in the next section. In Sect. 5 we consider the more general case of arbitrary metric on $X$, and the distinction between normal and conormal becomes essential there.

Remark. $\mathbb{D}+\mathrm{Q}(\mathrm{x})$ is the Dirac operator on the trivial 2-dimensional complex vector bundle over $X$ with compatible unitary connection defined by the function $q(x)$. So the change of $q_{t}$ with $t$ is equivalent to a change of the connection.

The boundary condition (3) is gauge invariant with respect to conjugation by $\mu$, while $D_{0}$ and $D_{1}$ are conjugate by $\mu$. So the operators $D_{0}, D_{1}$ with the same boundary condition (3) are isospectral, and the spectral flow of the family $D_{t}$ gives us a shift of the spectrum of $D_{t}$ when $t$ runs from 0 to 1 .

Theorem 1. The spectral flow of the family $\left(\mathrm{D}_{\mathrm{t}}\right), \mathrm{t} \in[0,1]$, with boundary condition (3) is equal to

$$
c_{m} \sum_{j=1}^{m} b_{j} \mu_{j}
$$

where $\mathrm{c}_{\mathrm{m}}$ is an integer constant depending on $\mathrm{m}$ only, $\mu_{\mathrm{j}}$ is the degree of the restriction of $\mu$ to $\partial \mathrm{X}_{\mathrm{j}}$,

$$
\mathrm{b}_{\mathrm{j}}=\left\{\begin{array}{ll}
1, & \text { if } \mathrm{B}<0 \text { on } \partial \mathrm{X}_{\mathrm{j}} \\
0, & \text { if } \mathrm{B}>0 \text { on } \partial \mathrm{X}_{\mathrm{j}}
\end{array} .\right.
$$

Here $\partial \mathrm{X}_{\mathrm{j}}$ are the connected components of the boundary of $\mathrm{X}$, equipped with an orientation in such a way that the pair (outward normal to $\partial \mathrm{X}_{\mathrm{j}}$, positive tangent vector to $\partial \mathrm{X}_{j}$ ) has positive orientation on the plane $\left(\chi^{1}, \chi^{2}\right)$ (see Fig. 1).

\footnotetext{
2 Another name for "locally elliptic boundary condition" is "Sapiro-Lopatinskii boundary condition".
} 


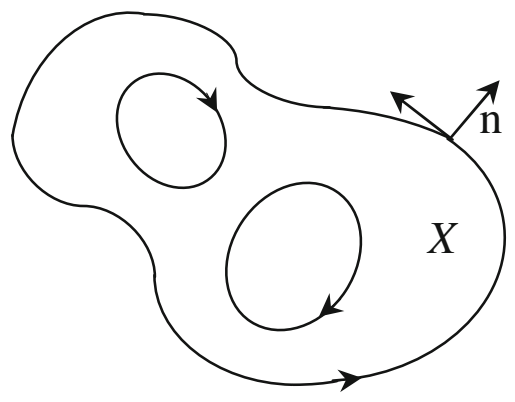

Fig. 1. The case of two holes

Note that since $B \neq 0$, it has definite sign at each boundary component $\partial X_{j}$, so the constants $b_{j}$ are correctly defined. The restriction of $\mu$ to the $j^{\text {th }}$ connected component of $\partial X$ gives us a map from the circle $\partial X_{j}$ to the circle $U(1) ; \mu_{j}$ is the degree of this map.

This theorem follows from a more general result which we formulate below. The generalization goes in two directions: (1) we allow arbitrary dimension for the unknown complex functions $\mathrm{u}^{-}, \mathrm{u}^{+},(2)$ we replace the Dirac operator by operators of a more general form. The value of $c_{2}$ is computed in Sect. 6 .

Remark. Boundary condition (3) coincides with the boundary condition of Berry and Mondragon for the "neutrino billiard" [3] up to replacement of B by $B^{-1}$. In physical terms, a one-parameter family of Dirac operators (2) describes the situation of a continuously varying magnetic field so that the following two conditions are fulfilled.

(1) the magnetic field at $t=1$ coincides with the magnetic field at $t=0$ all over the interior of $X$,

(2) the fluxes through the $j^{\text {th }}$ hole at $t=1$ and at $t=0$ differ by an integer number $\mu_{j}$ in the units of the flux quantum.

Suppose that $j=m$ corresponds to the outer boundary component and that $j=$ $1, \ldots, m-1$ enumerate the holes. Considering that $\mu_{m}=-\sum_{j=1}^{m-1} \mu_{j}$, we can reformulate Theorem 1 as follows. The spectral flow of the family of operators (2) with boundary condition (3) is equal to

$$
c_{m} \sum_{j=1}^{m-1}\left(b_{j}-b_{m}\right) \mu_{j} .
$$

Thus the variation of the magnetic field through the $j^{\text {th }}$ hole contributes to the value of the spectral flow with coefficient $c_{m}\left(b_{j}-b_{m}\right)$.

If the signs of B are the same on all boundary components, then the spectral flow is zero, no matter how the magnetic field is varied (if only conditions (1-2) above are fulfilled). In the contrary, if B takes positive values on some boundary component and negative values on another, then we can vary magnetic field so that the spectral flow does not vanish. 


\section{2N-dimensional Dirac operators}

Let $\mathrm{X}$ be as in the previous section. The standard $2 \mathrm{~N}$-dimensional Dirac operator has the form

$$
\mathbb{D}=-i\left(\sigma_{1} \partial_{1}+\sigma_{2} \partial_{2}\right), \text { where } \sigma_{1}=\left(\begin{array}{cc}
0 & \mathrm{I}_{\mathrm{N}} \\
\mathrm{I}_{\mathrm{N}} & 0
\end{array}\right), \sigma_{2}=\left(\begin{array}{cc}
0 & -i \mathrm{I}_{\mathrm{N}} \\
i \mathrm{I}_{\mathrm{N}} & 0
\end{array}\right),
$$

$I_{N}$ is the $N \times N$ unit matrix.

We will consider operators of the form $D=\mathbb{D}+Q(x)$ acting on spinor functions

$$
u=\left(\begin{array}{l}
u^{+} \\
u^{-}
\end{array}\right), \quad u^{ \pm}: X \rightarrow \mathbb{C}^{N},
$$

where $Q$ is a smooth map from $X$ to the space $H\left(\mathbb{C}^{2 N}\right.$ ) of complex self-adjoint (or, what is the same, Hermitian) $2 \mathrm{~N} \times 2 \mathrm{~N}$ matrices.

A self-adjoint local elliptic boundary condition for the operator $\mathbb{D}+Q$ has the form

$$
\text { in }(x) u^{+}=B(x) u^{-} \text {on } \partial X,
$$

where $B$ is a smooth map from $\partial X$ to the space of complex self-adjoint invertible $\mathrm{N} \times \mathrm{N}$ matrices, $n=\left(n_{1}, n_{2}\right)$ is the outward conormal to $\partial X$ at point $x$, and we identify $n$ with the complex number $n_{1}+i n_{2}$.

The equivalent representation of the boundary condition (6) is

$$
\left(i\left(n_{1} \sigma_{1}+n_{2} \sigma_{2}\right)+\left(\begin{array}{cc}
B^{-1} & 0 \\
0 & -B
\end{array}\right)\right) u=0 \text { on } \partial X .
$$

Theorem 2. Let $\mathrm{Q}_{\mathrm{t}}(\mathrm{x})$ be a continuous 1-parameter family of self-adjoint $2 \mathrm{~N} \times 2 \mathrm{~N}$ matrices smoothly dependent on $x \in X$ such that $\mathbb{D}+Q_{1}=\mu\left(\mathbb{D}+Q_{0}\right) \mu^{-1}$ for some smooth gauge transformation $\mu: \mathrm{X} \rightarrow \mathrm{U}(1)$. Let $\mathrm{B}$ be a smooth map from $\partial \mathrm{X}$ to the space of complex self-adjoint invertible $\mathrm{N} \times \mathrm{N}$ matrices. Then the spectral flow of the family $\left(\mathbb{D}+\mathrm{Q}_{\mathrm{t}}\right)$ with boundary condition (6) is equal to

$$
c_{m} \sum_{j=1}^{m} b_{j} \mu_{j},
$$

where $\mathrm{c}_{\mathrm{m}}$ is an integer constant depending on $\mathrm{m}$ only, $\mu_{\mathrm{j}}$ is the degree of the restriction of $\mu$ to the $j^{\text {th }}$ connected component $\partial X_{j}$ of the boundary, $b_{j}$ is the number of negative eigenvalues of $\mathrm{B}$ (counting multiplicities) on $\mathrm{\partial X}_{\mathfrak{j}}$ (this number is correctly defined due to the nondegeneracy of $\mathrm{B}$ ).

This result is a corollary of Theorem 3 from the next section.

\section{Dirac Type Operators}

Let $X$ be a compact planar domain bounded by $m$ smooth curves and equipped with a Riemannian metric $\mathrm{g}$ (which is not necessarily flat).

We call a first order formally self-adjoint operator $\mathrm{D}$ over $\mathrm{X}$ a Dirac type operator if its symbol has the form

$$
\rho=\left(\begin{array}{l}
\rho_{1} \\
\rho_{2}
\end{array}\right)=\Phi(x)\left(\begin{array}{c}
\sigma_{1} \\
\sigma_{2}
\end{array}\right)
$$


where $\Phi$ is a smooth map from $X$ to the group $\mathrm{GL}^{+}(2, \mathbb{R})$ of real invertible $2 \times 2$ matrices with positive determinant, and the matrices $\sigma_{1}, \sigma_{2}$ are defined by formula (4).

In other words, a Dirac type operator is an operator acting on spinor functions (5) and having the following form:

$$
\mathrm{D}=\mathrm{D}_{\Phi, \mathrm{Q}}=-i\left(\rho_{1}(x) \partial_{1}+\rho_{2}(x) \partial_{2}\right)+i R_{\Phi}(x)+\mathrm{Q}(x)
$$

where $Q$ is a smooth map from $X$ to the space $H\left(\mathbb{C}^{2 N}\right)$ of complex self-adjoint $2 N \times 2 N$ matrices,

$$
R_{\Phi}=\frac{1}{2}\left[\left(\rho_{1} \partial_{1}+\rho_{2} \partial_{2}\right)+\left(\rho_{1} \partial_{1}+\rho_{2} \partial_{2}\right)^{t}\right]
$$

(the superscript $t$ denotes the operation of taking the formal adjoint operator). More explicitly,

$$
\mathrm{R}_{\Phi}(x)=-\frac{1}{2}\left[\partial_{1}\left(\sqrt{g} \rho_{1}\right)+\partial_{2}\left(\sqrt{g} \rho_{2}\right)\right] \in \mathrm{H}\left(\mathbb{C}^{2 \mathrm{~N}}\right)
$$

where $\sqrt{g}=\sqrt{\operatorname{det}\left(g_{i j}\right)}$, the matrix $\left(g_{i j}\right)$ is inverse to the matrix $\left(g^{i j}\right)=\left(\left\langle d x^{i}, d x^{j}\right\rangle_{g}\right)$, $\sqrt{g} d x^{1} d x^{2}$ is the volume element on $X$ (of course, $g_{i j}, g^{i j}$, and $\sqrt{g}$ depend on $x$ ).

By $\mathcal{D}=\mathcal{D} x, g, N$ we denote the space of all operators having the form (9) for fixed $\mathrm{X}, \mathrm{g}, \mathrm{N}$. Note that a Dirac type operator (that is an element of $\mathcal{D} \mathrm{X}, \mathrm{g}, \mathrm{N}$ ) is uniquely defined by the pair $(\Phi, Q)$.

A self-adjoint elliptic local boundary condition for Dirac type operator (9) has the form

$$
\operatorname{in}^{\prime}(x) u^{+}=B(x) u^{-} \text {on } \partial X
$$

where $B$ is a smooth map from $\partial X$ to the space of complex self-adjoint invertible $\mathrm{N} \times \mathrm{N}$ matrices, the complex-valued function $n^{\prime}$ on $\partial X$ is defined by the formula $n^{\prime}=n_{1}^{\prime}+i n_{2}^{\prime}$ with $\left(n_{1}^{\prime}, n_{2}^{\prime}\right)=\left(n_{1}, n_{2}\right) \Phi$, where $n=\left(n_{1}, n_{2}\right)$ is the outward conormal to $\partial X$ at $x \in \partial X$. Recall that $n_{i}=\sum g_{i j} n^{j}$ for the components $\left(n^{j}\right)$ of the normal to the boundary.

Remark. Equation (10) is just another form of the equation

$$
i \rho^{+}(x, n(x)) u^{+}=B(x) u^{-} \text {on } \partial x
$$

where

$$
\rho(x, \xi)=\left(\begin{array}{cc}
0 & \rho^{-}(x, \xi) \\
\rho^{+}(x, \xi) & 0
\end{array}\right)
$$

denotes the symbol $\xi_{1} \rho_{1}(x)+\xi_{2} \rho_{2}(x)$ of the operator D in the direction of a covector $\xi=\left(\xi_{1}, \xi_{2}\right)$. Considering that in our case the operator $\rho^{+}(x, \xi)$ is scalar, and $\rho^{+}(x, n(x))=n^{\prime}(x) I_{N}$, the boundary condition (11) may be written in simplified form (10). 
By $\mathcal{B}=\mathcal{B}_{X, N}$ we denote the space of all smooth maps from $\partial X$ to the space of complex self-adjoint invertible $\mathrm{N} \times \mathrm{N}$ matrices.

Suppose that $\mathrm{D} \in \mathcal{D}, \mathrm{B} \in \mathcal{B}$. We will write ( $\mathrm{D}, \mathrm{B})$ for the operator (9) acting on the domain

$$
\left\{u \in C^{1}\left(X, \mathbb{C}^{2 N}\right): \text { restriction of } u \text { to } \partial X \text { satisfies boundary condition (10) }\right\},
$$

where $C^{1}\left(X, \mathbb{C}^{2 N}\right)$ is the space of continuously differentiable functions from $X$ to $\mathbb{C}^{2 N}$.

Such operators have the following properties:

1. For any $\mathrm{D} \in \mathcal{D}, \mathrm{B} \in \mathcal{B}$ the operator $(\mathrm{D}, \mathrm{B})$ is an (unbounded) essentially selfadjoint Fredholm operator, which has discrete real spectrum. All its eigenvectors are smooth functions. (Lemma 1, Sect. 10)

2. Suppose $Q_{t}(x)$ is continuous on $(t, x), D \in \mathcal{D}, B \in \mathcal{B}$. Then all operators from the family $\left(D+Q_{t}, B\right)$ have the same domain, and this family is norm continuous in $L^{2}\left(X, g ; \mathbb{C}^{2 N}\right)$. Therefore the spectral flow of the operator family $\left(D+Q_{t}, B\right)$ is well defined ([12], Prop. 2.2).

Now we can formulate the main result of the present paper:

Theorem 3. Suppose that $\mathrm{D} \in \mathcal{D}$ is a Dirac type operator (9), and let $\mathrm{B} \in \mathcal{B}$ define the boundary condition (10) for $\mathrm{D}$. Suppose that $\mathrm{Q}_{\mathrm{t}}(\mathrm{x})$ is a continuous 1-parameter family of self-adjoint $2 \mathrm{~N} \times 2 \mathrm{~N}$ matrices smoothly dependent on $\mathrm{x} \in \mathrm{X}$ such that $\mathrm{D}+\mathrm{Q}_{1}=\mu\left(\mathrm{D}+\mathrm{Q}_{0}\right) \mu^{-1}$ for some smooth gauge transformation $\mu: \mathrm{X} \rightarrow \mathrm{U}(1)$. Then

$$
\operatorname{sf}\left(D+Q_{t}, B\right)_{t \in[0,1]}=c_{m} \sum_{j=1}^{m} b_{j} \mu_{j}
$$

where $\mathrm{c}_{\mathrm{m}}$ is an integer constant depending on $\mathrm{m}$ only, $\mathrm{b}_{\mathrm{j}}$ is the number of negative eigenvalues of $\mathrm{B}$ (counting multiplicities) on $\partial \mathrm{X}_{\mathrm{j}}, \mu_{\mathrm{j}}$ is the degree of the restriction of $\mu$ to $\partial \mathrm{X}_{\mathfrak{j}}$, and $\partial \mathrm{X}$ is oriented as described in the statement of Theorem 1.

Note that constant $c_{m}$ in all Theorems 1-3 is the same.

Remark. Let $S$ be a spinor bundle over X. Suppose that $\langle\cdot, \cdot\rangle$ is a Hermitian metric on $S$ compatible with its spinor structure, and that $\nabla$ is a connection on $S$ compatible with its spinor structure and with the Levi-Civita connection on TX. The Dirac operator on $S$ in local coordinates has the form $\mathrm{D}=\mathrm{c}(v) \nabla_{v}+\mathrm{c}(w) \nabla_{\mathcal{w}}$, where $(v, w)$ is a positively oriented orthonormal basis in $T_{X} X$, and $c(v)$ denotes the action of a tangent vector $v$ on spinors.

The unitary skew-adjoint isomorphism $\mathrm{J}_{x}=\mathrm{c}(v) \mathrm{c}(w)$ of $\mathrm{S}_{\mathrm{x}}$ does not depend on the choice of a basis $(\nu, w)$ in $T_{\chi} X$ and defines the bundle decomposition $S=S^{+} \oplus S^{-}$, where $S^{ \pm}$are the subbundles of $S$ such that $S_{\chi}^{ \pm}$are the eigenspaces of $J_{\chi}$ corresponding to its eigenvalues $\mp i$. Due to the triviality of TX and of any complex bundle over $X$, we can fix some global positively oriented orthonormal basis field $(v(x), w(x))$ in TX and some trivialization of $S^{-}$. Let us extend the trivialization from $S^{-}$to $S$ so that the action of the tangent vectors on the spinors in this trivialization has the form

$$
c(v(x))=-i\left(\begin{array}{cc}
0 & \mathrm{I}_{\mathrm{N}} \\
\mathrm{I}_{\mathrm{N}} & 0
\end{array}\right), \quad c(w(x))=-i\left(\begin{array}{cc}
0 & -i \mathrm{I}_{\mathrm{N}} \\
i \mathrm{I}_{\mathrm{N}} & 0
\end{array}\right) .
$$


Then sections $u$ of the spinor bundle $S$ can be identified with the column vectors (5) of two functions $\mathrm{u}^{ \pm}: X \rightarrow \mathbb{C}^{\mathrm{N}}$, and the Dirac operator $\mathrm{D}$ acting on such column vectors can be written in the form $\mathrm{D}=-i\left(\rho_{1} \nabla_{1}+\rho_{2} \nabla_{2}\right)$. Here $\rho_{1}, \rho_{2}$ are defined by formula (8) with $\Phi(x)$ being the transition matrix: $(v, w)=\left(e_{1}, e_{2}\right) \Phi(x)$, where we denote by $e_{i}$ the vector (not the differential operator) $\partial_{i}$ to avoid misunderstanding.

So any Dirac operator over $X$ has the form (9) with $\Phi(x)$ satisfying the condition $\Phi(x) \Phi^{*}(x)=\left(g^{i j}(x)\right)$ and with $Q(x)$ a matrix of a very special kind. While allowing arbitrary metric $g$ and arbitrary $\mathrm{Q}(\mathrm{x})$ is important for some physical applications, considering Clifford multiplication which does not agree with the metric on $X$ (that is a matrix function $\Phi(x)$ which does not satisfy the condition $\left.\Phi(x) \Phi^{*}(x)=\left(g^{i j}(x)\right)\right)$ does not seem necessary. Nevertheless, we take care of this more general case because the proofs of our results crucially depend on it.

\section{The Case of One Hole}

Here we compute the spectral flow for the case when $X$ has just one hole $(m=2)$, and as a result find $c_{2}$.

Theorem 4. $c_{2}=1$.

Proof. By Theorem 3, the spectral flow does not depend on the geometry of X and on the choice of $\mathrm{D} \in \mathcal{D}$, so it is sufficient to consider only the case when the computation is as simple as possible. Let us take the annulus $X=\{(r, \varphi): 1 \leqslant r \leqslant 2\}$ in polar coordinates $(r, \varphi)$ on the plane, with the metric $d s^{2}=d r^{2}+d \varphi^{2}, N=1$,

$\mathrm{D}=-i\left(\begin{array}{cc}0 & \partial_{\mathrm{r}}-i \partial_{\varphi} \\ \partial_{\mathrm{r}}+i \partial_{\varphi} & 0\end{array}\right), \quad \mu=e^{i \varphi}, \quad Q_{t}=\left(\begin{array}{cc}0 & i t \\ -i t & 0\end{array}\right), \quad B=\left\{\begin{array}{cc}+1 & \text { at } r=1 \\ -1 & \text { at } r=2\end{array}\right.$.

We obtain the following system for the eigenvector $u$ and the eigenvalue $\lambda$ of $\left(\mathrm{D}+\mathrm{Q}_{\mathrm{t}}, \mathrm{B}\right)$ :

$$
\left\{\begin{array}{l}
\left(-i \partial_{r}+\partial_{\varphi}-i t\right) u^{+}=\lambda u^{-} \\
\left(-i \partial_{r}-\partial_{\varphi}+i t\right) u^{-}=\lambda u^{+} \\
u^{+}=i u^{-} \text {at } r=1,2
\end{array}\right.
$$

All the eigenvectors of $\left(D+Q_{t}, B\right)$ are smooth functions, so we can seek them in the form $u^{ \pm}(r, \varphi)=\sum_{k \in \mathbb{Z}} u_{k}^{ \pm}(r) e^{i k \varphi}$. Substituting it in the last system, we obtain

$$
\left\{\begin{array}{l}
\partial_{\mathrm{r}} u_{\mathrm{k}}^{+}-(k-t) u_{\mathrm{k}}^{+}-i \lambda u_{\mathrm{k}}^{-}=0 \\
\partial_{\mathrm{r}} u_{\mathrm{k}}^{-}+(k-t) u_{\mathrm{k}}^{-}-i \lambda u_{\mathrm{k}}^{+}=0 \\
u_{\mathrm{k}}^{+}=i u_{k}^{-} \text {at } r=1,2
\end{array}\right.
$$

Equivalently,

$$
\left\{\begin{array}{l}
\partial_{\mathrm{r}}\left(u_{\mathrm{k}}^{+}+i u_{\mathrm{k}}^{-}\right)=(\mathrm{k}-\mathrm{t}-\lambda)\left(\mathrm{u}_{\mathrm{k}}^{+}-i u_{\mathrm{k}}^{-}\right) \\
\partial_{\mathrm{r}}\left(\mathrm{u}_{\mathrm{k}}^{+}-i u_{\mathrm{k}}^{-}\right)=(\mathrm{k}-\mathrm{t}+\lambda)\left(\mathrm{u}_{\mathrm{k}}^{+}+i u_{\mathrm{k}}^{-}\right) \\
u_{\mathrm{k}}^{+}-i u_{\mathrm{k}}^{-}=0 \text { at } \mathrm{r}=1,2
\end{array}\right.
$$

and $\partial_{\mathrm{r}}^{2}\left(u_{\mathrm{k}}^{+}-i u_{\mathrm{k}}^{-}\right)=\left((k-t)^{2}-\lambda^{2}\right)\left(u_{\mathrm{k}}^{+}-i u_{\mathrm{k}}^{-}\right)$. So we have the following cases: 
- $\quad$ either $u_{\mathrm{k}}^{+}=u_{\mathrm{k}}^{-} \equiv 0$,

- $\quad$ or $k-t+\lambda=0, u_{k}^{-}=$const, $u_{k}^{+}=i u_{k}^{-}$,

- $\quad$ or $(k-t)^{2}-\lambda^{2}=-(\pi l)^{2}, l \in \mathbb{Z} \backslash\{0\}, u_{k}^{+}-i u_{k}^{-}=$const $\cdot\left(e^{\pi i l r}-e^{-\pi i l r)}\right)$.

Therefore the set

$$
\Lambda=\left\{(t, \lambda): \lambda \text { is an eigenvalue of }\left(D+Q_{t}, B\right)\right\}
$$

can be represented as the union $\Lambda=\Lambda_{1} \cup \Lambda_{2}$, where $\Lambda_{1}=\{(t, \lambda): \lambda-t \in \mathbb{Z}\}$ (with multiplicities 1 for all eigenvalues), $\Lambda_{2}$ is a subset of $\{(t, \lambda):|\lambda| \geqslant \pi\}$.

If $\lambda_{j}(t)$ are continuous functions from the interval $[0,1]$ to $\mathbb{R}$ such that $\lambda_{i}(t) \leqslant \lambda_{j}(t)$ for $i \leqslant j$ and $\Lambda \cap\{(t, \lambda): 0 \leqslant t \leqslant 1\}$ is the union of the graphs of functions $\lambda_{j}(t)$, then $\lambda_{j}(t)=j+t$ when $-3 \leqslant j \leqslant 2$ (up to a shift of the numeration). So

$$
\operatorname{sf}\left(\mathrm{D}+\mathrm{Q}_{\mathrm{t}}, \mathrm{B}\right)_{\mathrm{t} \in[0,1]}=1 \text {. }
$$

On the other hand, by Theorem 3,

$$
\text { sf }\left(D+Q_{t}, B\right)_{t \in[0,1]}=c_{2}\left(b_{1} \mu_{1}+b_{2} \mu_{2}\right)=c_{2} \mu_{2}=c_{2},
$$

where by $\partial X_{1}, \partial X_{2}$ we denote the inner and the outer boundary circles respectively. Therefore $c_{2}=1$.

\section{The Case of Several Holes}

In the case of $m>2$ such explicit computations as in the previous section are hardly possible, but from the very beginning of her work the author conjectured that $c_{m}=1$ for all $\mathrm{m}$; a partial motivation for this conjecture is provided in this section. After the preprint version [15] of this paper was distributed, M. I. Katsnelson and V. E. Nazaikinski posted preprint [11], in which they proved, in particular, this conjecture of the author.

Let us realize $X=X^{h}$ as $(m-1)$ identical annuli arranged along a line and connected by a band of the width $h$, with the corners smoothed out, as in Fig. 2.

Let us consider the process of continuous decreasing of the band's width from $h=1$ to $h=0$; we suppose that the annuli do not change in this process. Let us fix some function $\mu$ from $X^{1}$ to $U(1)$ and take $q_{t}=i t \mu^{-1}\left(\partial_{1} \mu+i \partial_{2} \mu\right)$. Restricting $\mu$ and $q_{t}$ to $X^{h}, 0<h \leqslant 1$, we obtain the operator (2) on $X^{h}$. Let us define the boundary condition by $B^{h}=-1$ on the inner part $\cup_{j<m} \partial X_{j}^{h}$ of $\partial X^{h}$ and $B^{h}=+1$ on the outer part $\partial X_{m}^{h}$ of $\partial X^{h}$.

By Theorem $1, \operatorname{sf}\left(\mathbb{D}+Q_{t}\right)=c_{m} \sum_{j<m} \mu_{j}$ does not depend on $h$. It is natural to suggest that the limit at $h \rightarrow+0$ of the (constant) spectral flow of the family $\left(\mathbb{D}+Q_{t}, B^{h}\right)$ for $X^{h}$ is equal to the spectral flow of $\left(\mathbb{D}+Q_{t}, B^{0}\right)$ for the "limit" domain $X^{0}$, which is the disjoint union of $\mathrm{m}-1$ annuli, and the "limit" boundary condition $\mathrm{B}^{0}=-1$ at the inner boundary and $\mathrm{B}^{0}=+1$ at the outer boundary of every annulus.

However, $\operatorname{sf}\left(\mathbb{D}+Q_{t}, B^{0}\right)$ for such union is the sum of $\operatorname{sf}\left(\mathbb{D}+Q_{t}, B^{0}\right)$ for the annuli, and hence is equal to $\sum_{j<m} c_{2} \mu_{j}=c_{2} \sum_{j<m} \mu_{j}$.

Therefore, if the assumption on the limiting behavior of the spectral flow is true, then $c_{m}=c_{2}$ for any $m>2$.

Another way to have a look at the general case is to fix the outer boundary and to increase the holes up to their merging, as in Fig. 3. Here we obtain the single annulus 


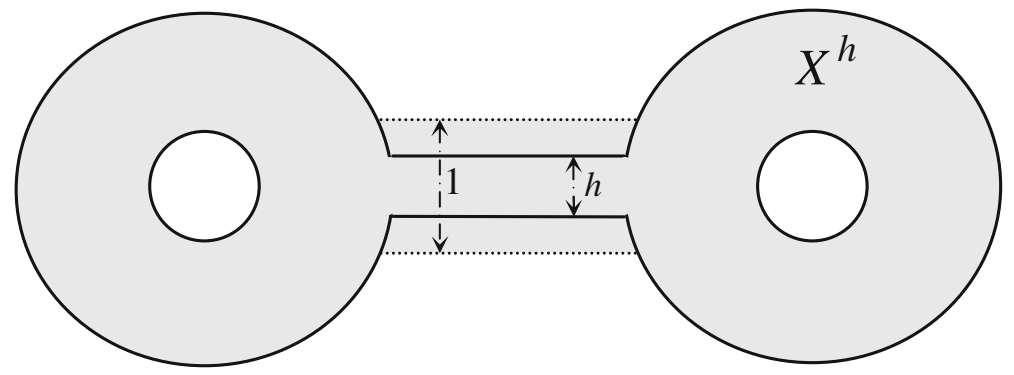

Fig. 2. Contracting of the connecting band
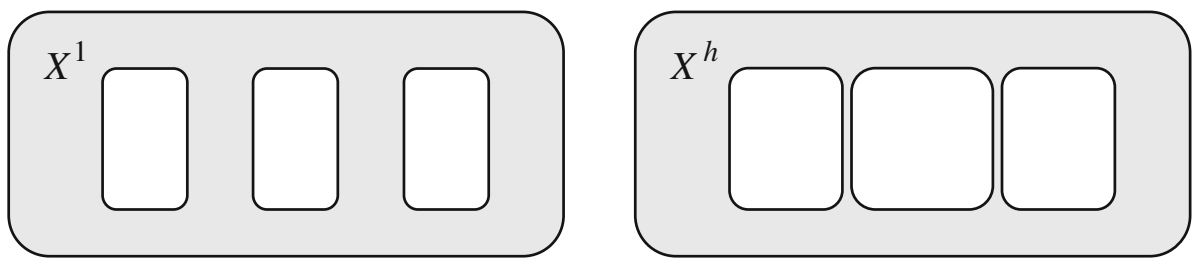

Fig. 3. Increasing of the holes

in the limit of $h=0$, and the same result $c_{m}=1$ if the passage to the limit will be justified.

Alternatively, we can combine these two methods to obtain arbitrary number $\mathrm{m}^{\prime}$, $1 \leqslant m^{\prime} \leqslant m-1$ of annuli in the end of the limit process, with the same result for $c_{m}$.

\section{General Case: First Order Elliptic Operators}

The results of the present paper are concerned only with the case when $X$ is a disk with holes. An easy modification of the proof gives us the analogue of this result for the case of a smooth compact oriented surface $X$ with nonempty boundary, with the only change being from $c_{m}$ to $c_{m, g}$, where $c_{m, g}$ is an integer constant depending on the number $m$ of boundary components of $X$ and on the genus $g$ of $X$. However, this still remains within a very restricted framework: all the operators $D_{t}$ are of Dirac type, both the symbol of $D_{t}$ and the boundary condition do not depend on $t$, and the conjugating gauge transformation is scalar.

In fact, this result can be extended to a much more general case. Namely, let $X$ be a smooth compact surface, let $\left(A_{t}\right)$ be a 1-parameter family of first order symmetric elliptic differential operators acting on sections of a unitary vector bundle $E$ over $X$, and suppose that $L_{t}$ is a subbundle of $\left.E\right|_{\partial x}$ which defines a self-adjoint elliptic local boundary condition for $A_{t}$ at any t. Suppose that $\left(A_{1}, L_{1}\right)$ is conjugate to $\left(A_{0}, L_{0}\right)$ by some gauge transformation $\mu$ (that is $\mu$ is a unitary isomorphism of $E$, not necessarily scalar). Then the operators $\left(A_{1}, L_{1}\right),\left(A_{0}, L_{0}\right)$ are isospectral, and there arises the natural question about the spectral flow of the family $\left(A_{t}, L_{t}\right)$. This question will be considered in a forthcoming paper [16]. In that paper we will prove that

$$
\operatorname{sf}\left(A_{t}, L_{t}\right)_{t \in[0,1]}=c_{m, g} \sum_{j=1}^{m} \varphi_{j},
$$


where $c_{m, g}$ is an integer depending on the number $m$ of boundary components of $X$ and on the genus $\mathrm{g}$ of $X, \varphi_{\mathrm{j}}$ is an integer determined in a canonical way by the restriction to the $j^{\text {th }}$ boundary component of the following data:

(1) the family $\left(\rho_{t}\right)$, where $\rho_{t}$ is the symbol of $A_{t}$;

(2) the family $\left(L_{t}\right)$ of boundary conditions;

(3) the gauge transformation $\mu$.

In particular, the spectral flow of $\left(A_{t}, L_{t}\right)$ does not depend on the choice of operators in the interior of $X$ but only on the symbol of the operators on the boundary.

Theorem 3 of the present paper fits into this general result as follows: $c_{m}=c_{m, 0}$, $\varphi_{j}=b_{j} \mu_{j}$. Recall that $\mu_{j}$ is determined by the restriction of $\mu$ to the $j^{\text {th }}$ boundary component of $X$, and $b_{j}$ is determined by the restrictions of the boundary condition and of the operator's symbol to the $j^{\text {th }}$ boundary component.

\section{The Spectral Flow for $\mathbf{N}=2$ in Terms of Condensed Matter Physics}

In this section we compare our boundary condition (6) with "the general boundary conditions for the Dirac equation" for 4-dimensional Dirac operators formulated by Akhmerov and Beenakker in [1]. After that, we give some computations for the spectral flow in terms of [1]. In particular, we show that the spectral flow vanishes in the case of time reversal symmetry (under the assumption of local ellipticity of the boundary problem).

In this section we will temporarily use the notations from [1] in their original form and will formulate our results in the same terms.

The long-wavelength and low-energy electronic excitations in graphene (a one-atomthick planar sheet of single carbon atoms that are densely packed in a honeycomb crystal lattice) considered in [1] are described by the Dirac equation $H \Psi=\varepsilon \Psi$ with Hamiltonian

$$
\mathrm{H}=v \tau_{0} \otimes(\boldsymbol{\sigma} \cdot \boldsymbol{p})
$$

acting on a four-component spinor wave function $\Psi=\left(\Psi_{A}, \Psi_{B}\right)$ (in our notations, $\Psi$ is a two-dimensional spinor function, $\Psi_{\mathrm{A}}=\mathrm{u}^{+}, \Psi_{\mathrm{B}}=\mathrm{u}^{-}, \mathrm{N}=2$ ). Here $v$ is the Fermi velocity, $\boldsymbol{p}=-i \hbar \nabla$ is the momentum operator, $\boldsymbol{\sigma} \cdot \boldsymbol{p}=-i \hbar\left(\sigma_{1} \nabla_{1}+\sigma_{2} \nabla_{2}\right)$, matrices $\tau_{i}, \sigma_{i}$ are Pauli matrices in valley space and sublattice space, respectively:

$$
\sigma_{0}=\left(\begin{array}{ll}
1 & 0 \\
0 & 1
\end{array}\right), \sigma_{1}=\left(\begin{array}{ll}
0 & 1 \\
1 & 0
\end{array}\right), \sigma_{2}=\left(\begin{array}{cc}
0 & -i \\
i & 0
\end{array}\right), \sigma_{3}=\left(\begin{array}{cc}
1 & 0 \\
0 & -1
\end{array}\right), \tau_{i}=\sigma_{i} .
$$

The general energy-independent boundary condition posed in [1] has the form

$$
\Psi=M \Psi \text { on the boundary, }
$$

where $M$ is a self-adjoint unitary $4 \times 4$ matrix depending on the point $x \in \partial X$ and anticommuting with the current operator $v \tau_{0} \otimes\left(\boldsymbol{\sigma} \cdot \boldsymbol{n}_{\mathrm{B}}\right)$. Here $\boldsymbol{n}_{\mathrm{B}}$ is the outward normal to the boundary of $X$ at $\chi$, so $\boldsymbol{n}_{\mathrm{B}}=\left(\mathrm{n}_{1}, \mathrm{n}_{2}\right)$ in our previous notations, and $\boldsymbol{\sigma} \cdot \boldsymbol{n}_{\mathrm{B}}=$ $n_{1} \sigma_{1}+n_{2} \sigma_{2}$.

Let us compare (13) with our boundary condition (6).

At first note that the condition " $M$ is a self-adjoint unitary matrix anticommuting with the current operator" means nothing but the condition of self-adjointness of the boundary problem (13). The authors of [1] do not require local ellipticity of the boundary condition; however, in the absence of local ellipticity the spectrum of the operator is not expected to be discrete. The boundary condition (13) is both locally elliptic and 
self-adjoint if and only if the matrix function $M(x)$ can be represented by a formula of the form

$$
M=I_{2 N}-2\left(\begin{array}{cc}
I_{N}+B^{2} & 0 \\
0 & I_{N}+B^{2}
\end{array}\right)^{-1}\left(\begin{array}{cc}
I_{N} & i \bar{n} B \\
-i n B & B^{2}
\end{array}\right)
$$

for some complex self-adjoint invertible $\mathrm{N} \times \mathrm{N}$ matrix function $\mathrm{B}(\mathrm{x})$. For such a matrix function $M$ the boundary condition (13) is equivalent to our boundary condition (7).

The set of all possible self-adjoint unitary $4 \times 4$ matrices anticommuting with the current operator is parameterized in [1] by the following 4-parameter family:

$$
M=\sin \Lambda \tau_{0} \otimes\left(\boldsymbol{n}_{1} \cdot \boldsymbol{\sigma}\right)+\cos \Lambda(\boldsymbol{v} \cdot \boldsymbol{\tau}) \otimes\left(\boldsymbol{n}_{2} \cdot \boldsymbol{\sigma}\right),
$$

where $\Lambda(x)$ is the "mixing angle", $\boldsymbol{v}(x), \boldsymbol{n}_{1}(x), \boldsymbol{n}_{2}(x)$ are unit vectors in $\mathbb{R}^{3}=$ $\left\{\left(x^{1}, x^{2}, x^{3}\right)\right\}$ such that $\boldsymbol{n}_{1}$ and $\boldsymbol{n}_{2}$ are mutually orthogonal and also orthogonal to the boundary normal $\boldsymbol{n}_{\mathrm{B}}(x),(\boldsymbol{v} \cdot \boldsymbol{\tau})=\sum_{i=1}^{3} v^{i} \tau_{i}$, and $\left(\boldsymbol{n}_{j} \cdot \boldsymbol{\sigma}\right)$ are defined analogously. Now we give a description of the ellipticity of the boundary problem (13) in terms of $\Lambda$, $\boldsymbol{v}, \boldsymbol{n}_{1}, \boldsymbol{n}_{2}$, and compute $b_{j}$ as functions of these parameters.

From now on we will suppose that the frame $\left(\boldsymbol{n}_{\mathrm{B}}, \boldsymbol{n}_{1}, \boldsymbol{n}_{2}\right)$ is positively oriented in $\mathbb{R}^{3}$, that is its orientation coincides with the orientation of the frame $\left(e_{1}, e_{2}, e_{3}\right)$ of basis coordinate vectors. This is possible because parameters $\left(\Lambda, \boldsymbol{n}_{1}, \boldsymbol{n}_{2}, \boldsymbol{v}\right)$ and $\left(-\Lambda,-\boldsymbol{n}_{1}, \boldsymbol{n}_{2}, \boldsymbol{v}\right)$ give us the same matrix $M$, so in the case of a negatively oriented frame $\left(\boldsymbol{n}_{\mathrm{B}}, \boldsymbol{n}_{1}, \boldsymbol{n}_{2}\right)$ we can change the signs of $\boldsymbol{n}_{1}$ and $\Lambda$ simultaneously to obtain the positive orientation of the frame.

Let $\varphi(x)$ be a function from the boundary to the circle $\mathbb{R} \bmod 2 \pi$ such that $\boldsymbol{n}_{2}=$ $\sin \varphi \cdot \eta+\cos \varphi \cdot e_{3}$, where $e_{3}$ is the unit vector in $\mathbb{R}^{3}$ in the direction of $\chi^{3}, \eta(x)$ is the unit tangent vector to the boundary at $x \in \partial X$ such that the pair $\left(\boldsymbol{n}_{\mathrm{B}}(x), \eta(x)\right)$ has the positive orientation on the plane $\left(\chi^{1}, x^{2}\right)$. Then $\boldsymbol{n}_{1}=\cos \varphi \cdot \eta-\sin \varphi \cdot e_{3}$, and $M$ is determined by the triple $(\Lambda, \varphi, v)$.

Proposition 1. The boundary condition (13) is locally elliptic for the Dirac operator (12) if and only if $\Lambda+\varphi \neq 0(\bmod \pi)$ and $\Lambda-\varphi \neq 0(\bmod \pi)$ for any $x \in \partial X$.

In other words, the boundary condition is not locally elliptic if and only if $\boldsymbol{n}_{2}=$ $\pm \sin \Lambda \eta \pm \cos \Lambda e_{3}$ for some $x \in \partial X$ and for some combination of signs \pm .

Proposition 2. If the boundary condition (13) is locally elliptic for the Dirac operator (12) then it is equivalent to the boundary condition

$$
\operatorname{in}(x) \Psi_{A}=B(x) \Psi_{B}
$$

with the matrix $\mathrm{B}(\mathrm{x})$ defined as follows:

$$
\begin{aligned}
& B=\beta_{+} P_{+}+\beta_{-} P_{-}, \text {where } P_{ \pm}=\frac{\tau_{0} \pm(\boldsymbol{v} \cdot \boldsymbol{\tau})}{2}, \\
& \beta_{+}=\cot \frac{\Lambda+\varphi}{2}, \beta_{-}=\tan \frac{\Lambda-\varphi}{2} .
\end{aligned}
$$

Here $\beta_{ \pm}$are the eigenvalues of $\mathrm{B}, \mathrm{P}_{ \pm}$are the orthogonal projections on the invariant subspaces of $\mathrm{B}$ corresponding to the eigenvalues $\beta_{ \pm}$.

These propositions will be proved at the end of the section. 
Corollary 1. Let $\mathrm{Q}_{\mathrm{t}}(\mathrm{x})$ be a continuous 1-parameter family of self-adjoint $4 \times 4$ matrices smoothly dependent on $\mathrm{x} \in \mathrm{X}$ such that $\mathrm{H}+\mathrm{Q}_{1}=\mu\left(\mathrm{H}+\mathrm{Q}_{0}\right) \mu^{-1}$ for some smooth gauge transformation $\mu: \mathrm{X} \rightarrow \mathrm{U}(1)$. Suppose that the boundary condition (13) is locally elliptic for the Dirac operator (12). Then the spectral flow of the family $\left(\mathrm{H}+\mathrm{Q}_{\mathrm{t}}\right)$ with this boundary condition is described by the formulae

$$
\operatorname{sf}\left(H+Q_{t}, M\right)_{t \in[0,1]}=c_{m} \sum_{j=1}^{m} b_{j} \mu_{j}
$$

where $\mathrm{c}_{\mathrm{m}}, \mu_{\mathrm{j}}$ are as in Theorem $2, \mathrm{~b}_{\mathrm{j}}$ depends only on the values of $\Lambda, \varphi$ on $\mathrm{j}^{\mathrm{th}}$ boundary component:

$$
b_{j}=\left\{\begin{array}{c}
0, \text { if both } \Lambda+\varphi, \Lambda-\varphi \text { belong to the interval }(0, \pi) \\
2, \text { if both } \Lambda+\varphi, \Lambda-\varphi \text { belong to the interval }(\pi, 2 \pi) \\
1, \text { if one of } \Lambda+\varphi, \Lambda-\varphi \text { belongs to the interval }(0, \pi) \\
\text { and another to the interval }(\pi, 2 \pi) .
\end{array}\right.
$$

Proof. This follows immediately from Theorem 2 and Proposition 2.

Let us inspect closer the case of time reversal symmetry. The time reversal operator in the valley isotropic representation is

$$
\mathrm{T}=-\left(\tau_{2} \otimes \sigma_{2}\right) \mathcal{C}
$$

with $\mathcal{C}$ the operator of complex conjugation [1]. The boundary condition preserves time reversal symmetry if $M$ commutes with $T$. This implies that the mixing angle $\Lambda \equiv 0$ [1]. By Proposition 1, in this case boundary problem (13) is locally elliptic if and only if $\boldsymbol{n}_{2}(x)$ is not vertical for all $x \in \partial X$. If this is fulfilled then Corollary 1 allows us to compute the spectral flow regardless of other parameters:

Corollary 2 (The case of time reversal symmetry). Let $\mathrm{Q}_{\mathrm{t}}(\mathrm{x}), \mathrm{t} \in[0,1]$ be a continuous 1-parameter family of self-adjoint $2 \mathrm{~N} \times 2 \mathrm{~N}$ matrices smoothly dependent on $\mathrm{x} \in \mathrm{X}$ such that $\mathrm{H}+\mathrm{Q}_{1}=\mu\left(\mathrm{H}+\mathrm{Q}_{0}\right) \mu^{-1}$ for some smooth gauge transformation $\mu: \mathrm{X} \rightarrow \mathrm{U}(1)$. Suppose that boundary condition is defined by formulas (13), (14) with $\Lambda \equiv 0$, and that for any $\mathrm{x} \in \partial \mathrm{X}$ vector $\boldsymbol{n}_{2}(\mathrm{x})$ is not vertical. Then the spectral flow of the family $\left(\mathrm{H}+\mathrm{Q}_{\mathrm{t}}\right)$ is zero.

Proof. By Corollary $1, b_{j}=1$ for all $j$. So we obtain

$$
\operatorname{sf}\left(H+Q_{t}\right)_{t \in[0,1]}=c_{m} \sum_{j=1}^{m} b_{j} \mu_{j}=c_{m} \sum_{j=1}^{m} \mu_{j}=0 .
$$

Remark. Corollary 2 can be proved by other means as well, without use of Corollary 1 and of formula (14) but using Theorem 2 directly. Namely, let $M \Psi=\Psi$ be a locally elliptic boundary condition for the Dirac operator (12) such that $\mathrm{TMT}^{-1}=M$. First note that the spectral flow of the family $\left(H+Q_{t}\right)$ is independent of the choice of connection $\nabla$, so we can assume that $\nabla_{i}=\partial_{i}$. With this choice of connection, we have $\mathrm{THT}^{-1}=\mathrm{H}$. Let $\mathrm{Q}_{\mathrm{t}}^{\prime}(\mathrm{x})=\mathrm{TQ}_{\mathrm{t}}(\mathrm{x}) \mathrm{T}^{-1}$, then

$$
\begin{aligned}
\mathrm{Q}_{1}^{\prime}-\mathrm{Q}_{0}^{\prime} & =\mathrm{T}\left(\mathrm{Q}_{1}-\mathrm{Q}_{0}\right) \mathrm{T}^{-1}=\mathrm{T}\left(\mu \mathrm{H} \mu^{-1}-\mathrm{H}\right) \mathrm{T}^{-1} \\
& =\mu^{-1} \mathrm{THT}^{-1} \mu-\mathrm{THT}^{-1}=\mu^{\prime} \mathrm{H}^{\prime-1}-\mathrm{H},
\end{aligned}
$$


where $\mu^{\prime}=\mu^{-1}$. By Theorem 2,

$$
\operatorname{sf}\left(H+Q_{t}^{\prime}, M\right)=c_{m} \sum b_{j} \mu_{j}^{\prime}=-c_{m} \sum b_{j} \mu_{j}=-\operatorname{sf}\left(H+Q_{t}, M\right),
$$

where by $\mathrm{sf}\left(\mathrm{H}+\mathrm{Q}_{\mathrm{t}}, \mathrm{M}\right)$ we denote the spectral flow of the family $\left(H+\mathrm{Q}_{t}\right)$ with boundary condition $M \Psi=\Psi$. If $M$ commutes with $T$ then

$$
\operatorname{sf}\left(T\left(H+Q_{t}\right) T^{-1}, T M T^{-1}\right)=s f\left(H+Q_{t}^{\prime}, M\right)=-s f\left(H+Q_{t}, M\right) .
$$

In Sect. 11 we prove the conjugacy invariance of the spectral flow under unitary isomorphisms of $\mathrm{L}^{2}\left(X, g ; \mathbb{C}^{2 N}\right)$. Even though $\mathrm{T}$ is an antilinear isomorphism of the Hilbert space $\mathrm{L}^{2}\left(X, g ; \mathbb{C}^{4}\right)$, the spectral flow still remains invariant under conjugation by $\mathrm{T}$. This can be proved using the uniqueness property of the spectral flow in the same manner as Property (P4) in Sect. 11, taking into account that conjugation by $\mathrm{T}$ preserves selfadjointness of operators in $L^{2}\left(X, g ; \mathbb{C}^{4}\right)$. Thus $s f\left(T\left(H+Q_{t}\right) T^{-1}, T M T^{-1}\right)$ coincides with $\mathrm{sf}\left(\mathrm{H}+\mathrm{Q}_{\mathrm{t}}, M\right)$. Together with (15), this implies $\mathrm{sf}\left(\mathrm{H}+\mathrm{Q}_{\mathrm{t}}, M\right)=0$.

Proof of Proposition 1. By $S=S(x)$ we denote the matrix $(\boldsymbol{v} \cdot \boldsymbol{\tau})=\sum_{i=1}^{3} v^{i} \tau_{i}$. In our notations,

$$
\begin{aligned}
M & =\sin \Lambda \cdot \tau_{0} \otimes\left(\begin{array}{cc}
-\sin \varphi & -i \bar{n} \cos \varphi \\
i n \cos \varphi & \sin \varphi
\end{array}\right)+\cos \Lambda \cdot S \otimes\left(\begin{array}{cc}
\cos \varphi & -i \bar{n} \sin \varphi \\
i n \sin \varphi & -\cos \varphi
\end{array}\right) \\
& =\left(\begin{array}{cc}
S_{1} & -i \bar{n} S_{2} \\
i n S_{2} & -S_{1}
\end{array}\right),
\end{aligned}
$$

where $S_{1}=-\sin \varphi \sin \Lambda \cdot I+\cos \varphi \cos \Lambda \cdot S, S_{2}=\cos \varphi \sin \Lambda \cdot I+\sin \varphi \cos \Lambda \cdot S$, $\mathrm{I}=\mathrm{I}_{2}$ is the $2 \times 2$ identity matrix. Note that $S^{2}=\mathrm{I}$ for any $\boldsymbol{v}, S_{1}^{2}+S_{2}^{2}=\mathrm{I}$ for any $\boldsymbol{v}$, $\varphi, \Lambda$.

The boundary condition $M \Psi=\Psi$ is equivalent to the following system:

$$
\left\{\begin{array}{rl}
-i \bar{n} S_{2} u^{-} & =\left(I-S_{1}\right) u^{+} \\
i n S_{2} u^{+} & =\left(I+S_{1}\right) u^{-}
\end{array} .\right.
$$

This boundary problem is locally elliptic for the operator (12) if the linear space of the solutions of this system has intersection zero with both spaces $\left\{u^{+}=0\right\}$ and $\left\{u^{-}=0\right\}$. This condition is equivalent to the invertibility of $S_{2}$. The matrix $S$ has eigenvalues \pm 1 , so $S_{2}$ has the eigenvalues $\cos \varphi \sin \Lambda \pm \sin \varphi \cos \Lambda=\sin (\Lambda \pm \varphi)$. Both eigenvalues of $S_{2}$ are nonzero if and only if $\Lambda \pm \varphi \neq 0(\bmod \pi)$. This completes the proof.

Proof of Proposition 2. From (16) we have $B=S_{2}^{-1}\left(I+S_{1}\right)$. Taking into account the identity $S^{2}=I$, we obtain

$$
\begin{aligned}
S_{2}^{-1} & =\left(\cos ^{2} \varphi \sin ^{2} \Lambda-\sin ^{2} \varphi \cos ^{2} \Lambda\right)^{-1}(\cos \varphi \sin \Lambda \cdot I-\sin \varphi \cos \Lambda \cdot S) \\
S_{2}^{-1}\left(I+S_{1}\right) & =\left(\cos ^{2} \varphi \sin ^{2} \Lambda-\sin ^{2} \varphi \cos ^{2} \Lambda\right)^{-1}(\sin \Lambda-\sin \varphi)(\cos \varphi \cdot I+\cos \Lambda \cdot S) \\
& =\frac{\sin \Lambda-\sin \varphi}{\sin (\Lambda+\varphi) \sin (\Lambda-\varphi)}(\cos \varphi \cdot I+\cos \Lambda \cdot S) .
\end{aligned}
$$

The eigenvalues of $S$ are \pm 1 , so the eigenvalues of $B$ are equal to

$$
\beta_{ \pm}=\frac{\sin \Lambda-\sin \varphi}{\sin (\Lambda+\varphi) \sin (\Lambda-\varphi)}(\cos \varphi \pm \cos \Lambda) .
$$


From the last two formulas we have

$$
\begin{aligned}
\mathrm{B} & =\frac{\sin \Lambda-\sin \varphi}{\sin (\Lambda+\varphi) \sin (\Lambda-\varphi)}\left((\cos \varphi+\cos \Lambda) \frac{\mathrm{I}+\mathrm{S}}{2}+(\cos \varphi-\cos \Lambda) \frac{\mathrm{I}-\mathrm{S}}{2}\right) \\
& =\beta_{+} \frac{\mathrm{I}+\mathrm{S}}{2}+\beta_{-} \frac{\mathrm{I}-\mathrm{S}}{2}=\beta_{+} \mathrm{P}_{+}+\beta_{-} \mathrm{P}_{-} .
\end{aligned}
$$

We can simplify (17) using sum-to-product trigonometric identities:

$$
\begin{aligned}
\frac{\sin \Lambda-\sin \varphi}{\sin (\Lambda+\varphi) \sin (\Lambda-\varphi)} & =\frac{2 \sin \frac{\Lambda-\varphi}{2} \cos \frac{\Lambda+\varphi}{2}}{\left(2 \sin \frac{\Lambda+\varphi}{2} \cos \frac{\Lambda+\varphi}{2}\right)\left(2 \sin \frac{\Lambda-\varphi}{2} \cos \frac{\Lambda-\varphi}{2}\right)} \\
& =\left(2 \sin \frac{\Lambda+\varphi}{2} \cos \frac{\Lambda-\varphi}{2}\right)^{-1},
\end{aligned}
$$

$\cos \varphi+\cos \Lambda=2 \cos \frac{\Lambda+\varphi}{2} \cos \frac{\Lambda-\varphi}{2}, \quad \cos \varphi-\cos \Lambda=2 \sin \frac{\Lambda+\varphi}{2} \sin \frac{\Lambda-\varphi}{2}$.

Substituting this in (17), we obtain

$$
\beta_{+}=\cot \frac{\Lambda+\varphi}{2}, \quad \beta_{-}=\tan \frac{\Lambda-\varphi}{2} .
$$

This completes the proof.

\section{Part II Proof of Theorem 3}

Note that for $\mathrm{D}^{\prime}=\mathrm{D}+\mathrm{Q}_{0}, \mathrm{Q}_{\mathrm{t}}^{\prime}=\mathrm{Q}_{\mathrm{t}}-\mathrm{Q}_{0}$, we have $\mathrm{D}+\mathrm{Q}_{\mathrm{t}}=\mathrm{D}^{\prime}+\mathrm{Q}_{\mathrm{t}}^{\prime}$ with $\mathrm{Q}_{0}^{\prime}=0$. For this reason, in the proof we will restrict ourselves to the families $\mathrm{Q}_{t}$ with $\mathrm{Q}_{0}=0$.

\section{Two Technical Lemmas}

First of all, we need to give some technical details. The reader interested only in the ideas behind the proof can go directly to the next section.

Suppose $\mathrm{D} \in \mathcal{D}, \mathrm{B} \in \mathcal{B}$. We will write $(\mathrm{D}, \mathrm{B})$ for the operator $\mathrm{D}$ acting on the following domain:

$$
\begin{aligned}
& \operatorname{domain}(\mathrm{D}, \mathrm{B}) \\
& \quad=\left\{\mathrm{u} \in \mathrm{L}_{1}^{2}\left(\mathrm{X} ; \mathbb{C}^{2 \mathrm{~N}}\right): \text { restriction of } \mathrm{u} \text { to } \partial \mathrm{X} \text { satisfies boundary condition }(10)\right\} .
\end{aligned}
$$

Here $\mathrm{L}_{1}^{2}\left(X ; \mathbb{C}^{2 N}\right)$ is the first Sobolev space; its elements are functions $u \in \mathrm{L}^{2}\left(X ; \mathbb{C}^{2 N}\right)$ such that $\partial_{1} u, \partial_{2} u \in L^{2}\left(X ; \mathbb{C}^{2 N}\right)$. Strictly speaking, we use here not the restriction in the usual sense (trace map $\left.u \mapsto u\right|_{\partial X}$ ) but the extension by continuity of the trace map $C^{\infty}\left(X ; \mathbb{C}^{2 N}\right) \rightarrow C^{\infty}\left(\partial X ; \mathbb{C}^{2 N}\right)$ to the bounded linear map from $L_{1}^{2}\left(X ; \mathbb{C}^{2 N}\right)$ to $\mathrm{L}_{1 / 2}^{2}\left(\partial \mathrm{X} ; \mathbb{C}^{2 \mathrm{~N}}\right)[7]$.

Note that the operator $(\mathrm{D}, \mathrm{B})$ defined here is the closure of the operator $(\mathrm{D}, \mathrm{B})$ defined in Sect. 5 (see [7], Prop. 2.9). The use of non-closed operators in the first part of the paper is explained by our intention to avoid the introduction of Sobolev spaces and of the extension of the trace map as long as possible. Due to the following lemma, these two definitions give us operators with the same eigenvectors, so the slight abuse of notation does not cause any troubles. 
Lemma 1. For any $\mathrm{D} \in \mathcal{D}, \mathrm{B} \in \mathcal{B}$ the operator $(\mathrm{D}, \mathrm{B})$ is a (unbounded) closed selfadjoint Fredholm operator on $\mathrm{L}^{2}\left(\mathrm{X}, \mathrm{g} ; \mathbb{C}^{2 \mathrm{~N}}\right)$, which has the discrete real spectrum. Moreover, all its eigenvectors are smooth functions.

Proof. Let $\mathrm{B}$ be a smooth function from $\partial \mathrm{X}$ to $\mathrm{GL}(\mathrm{N}, \mathbb{C})$. Then for any $\mathrm{D} \in \mathcal{D}, \lambda \in \mathbb{C}$ boundary condition (10) satisfies the Sapiro-Lopatinskii condition for $D-\lambda$ : the intersections of the subspace $\left\{u: i n^{\prime}(x) u^{+}=B(x) u^{-}\right\} \subset \mathbb{C}^{2 N}$ both with $\left\{u: u^{-}=0\right\}$ and with $\left\{\mathfrak{u}: \mathrm{u}^{+}=0\right\}$ are zero at any $\mathrm{x} \in \partial \mathrm{X}$. By Proposition 2.9 from [7], condition (10) is a strongly regular boundary condition for $D$, so all eigenvectors of $(D, B)$ in $\mathrm{L}^{2}\left(\mathrm{X}, \mathrm{g} ; \mathbb{C}^{2 \mathrm{~N}}\right)$ are smooth functions. By the same proposition, $(\mathrm{D}-\lambda, \mathrm{B})$ is a closed Fredholm operator for any $\lambda \in \mathbb{C}$, so the spectrum of $(D, B)$ is discrete.

For any $u, w \in \mathrm{L}_{1}^{2}\left(\mathrm{X}, \mathrm{g} ; \mathbb{C}^{2 \mathrm{~N}}\right)$ we have

$$
\begin{aligned}
& \langle\mathrm{Du}, w\rangle_{\mathrm{L}^{2}}-\langle\mathrm{u}, \mathrm{D} w\rangle_{\mathrm{L}^{2}}=\int_{\mathrm{X}}(\langle\mathrm{Du}, w\rangle-\langle\mathrm{u}, \mathrm{D} w\rangle) \sqrt{\mathrm{g}} \mathrm{d} x^{1} \mathrm{~d} x^{2} \\
& =-i \int_{X}\left(\partial_{1}\left\langle\sqrt{g} \rho_{1} u, w\right\rangle+\partial_{2}\left\langle\sqrt{g} \rho_{2} u, w\right\rangle\right) d x^{1} d x^{2} \\
& =-i \int_{X} d\left(\left\langle\sqrt{g} \rho_{1} u, w\right\rangle d x^{2}-\left\langle\sqrt{g} \rho_{2} u, w\right\rangle d x^{1}\right) \\
& =-i \int_{\partial x} \sqrt{g}\left(\left\langle\rho_{1} u, w\right\rangle d x^{2}-\left\langle\rho_{2} u, w\right\rangle d x^{1}\right) \\
& =-i \int_{\partial x}\left\langle\left(n_{1} \rho_{1}+n_{2} \rho_{2}\right) u, w\right\rangle \sqrt{g} d s \\
& =-\int_{\partial x}\left\langle\left(\begin{array}{l}
i \bar{n}^{\prime} u^{-} \\
i n^{\prime} u^{+}
\end{array}\right),\left(\begin{array}{l}
w^{+} \\
w^{-}
\end{array}\right)\right\rangle \sqrt{g} d s \\
& =\int_{\partial x}\left(\left\langle u^{-}, i n^{\prime} w^{+}\right\rangle-\left\langle i n^{\prime} u^{+}, w^{-}\right\rangle\right) \sqrt{g} d s,
\end{aligned}
$$

where ds is the length element on $\partial X$. So for any $u, w \in$ domain(D, B),

$$
\langle\mathrm{Du}, w\rangle_{\mathrm{L}^{2}}-\langle\mathrm{u}, \mathrm{D} w\rangle_{\mathrm{L}^{2}}=\int_{\partial \mathrm{X}}\left\langle\mathrm{u}^{-},\left(\mathrm{B}-\mathrm{B}^{*}\right) w^{-}\right\rangle \sqrt{\mathrm{g}} \mathrm{ds},
$$

and the operator $(D, B)$ is symmetric on $L^{2}\left(X, g ; \mathbb{C}^{2 N}\right)$ if and only if $B(x)$ is self-adjoint at any $x$.

Let now $w \in \operatorname{domain}(\mathrm{D}, \mathrm{B})^{*}$. By Proposition 2.9 from [7], domain $(\mathrm{D}, \mathrm{B})^{*}$ is contained in $\mathrm{L}_{1}^{2}\left(\mathrm{X}, \mathrm{g} ; \mathbb{C}^{2 \mathrm{~N}}\right)$, so we can use the computation above:

$$
\langle\mathrm{Du}, w\rangle_{\mathrm{L}^{2}}-\langle\mathrm{u}, \mathrm{D} w\rangle_{\mathrm{L}^{2}}=\int_{\partial \mathrm{x}}\left\langle\mathrm{u}^{-},\left(\mathrm{in}^{\prime} w^{+}-\mathrm{B} w^{-}\right)\right\rangle \sqrt{\mathrm{g}} \mathrm{d} s
$$

for any $\mathrm{u} \in \operatorname{domain}(\mathrm{D}, \mathrm{B})$. Therefore, $\mathrm{in}^{\prime} w^{+}-\left.\mathrm{B} w^{-}\right|_{\partial \mathrm{x}}=0$ for any $w \in$ domain $(D, B)^{*}$, hence domain $(D, B)^{*}=\operatorname{domain}(D, B)$, and $(D, B)$ is self-adjoint on $L^{2}\left(X, g ; \mathbb{C}^{2 N}\right)$. All eigenvalues of a self-adjoint operator are real. This completes the proof.

In the statement of Theorem 3 we used only norm continuous paths of operators with fixed domain. But for the proof of Theorem 3 we have to deal with paths of a 
more general kind, when neither the symbol of the operator nor the boundary condition are fixed any more. The paths we need for the proof are not norm continuous but only graph continuous (note that by Proposition 2.2 from [12] any norm continuous path is graph continuous as well). So further we will use the graph topology on the space of closed densely defined self-adjoint operators on a separable Hilbert space $\mathrm{H}$ (in our case $\left.\mathrm{H}=\mathrm{L}^{2}\left(X, \mathrm{~g} ; \mathbb{C}^{2 \mathrm{~N}}\right)\right)$.

There are various definitions of the graph distance, all of which give the same graph topology [6]. One can take $d_{G}\left(A, A^{\prime}\right)=\left\|(A+i I)^{-1}-\left(A^{\prime}+i I\right)^{-1}\right\|$, or alternatively $\mathrm{d}_{\mathrm{G}}\left(A, A^{\prime}\right)=\left\|\mathrm{P}_{\mathrm{A}}-\mathrm{P}_{\mathrm{A}^{\prime}}\right\|$, where $\mathrm{P}_{\mathrm{A}}, \mathrm{P}_{\mathrm{A}^{\prime}}$ are the orthogonal projections of $\mathrm{H} \times \mathrm{H}$ onto the graphs of $A, A^{\prime}$ respectively.

Let us introduce the following metrics in $\mathcal{D}$ and $\mathcal{B}$ :

$$
\begin{aligned}
& d\left(D_{\Phi, Q}, D_{\Phi^{\prime}, Q^{\prime}}\right)=\left\|Q-Q^{\prime}\right\|_{C(X)}+\left\|\Phi-\Phi^{\prime}\right\|_{C^{1}(X)} \\
& =\max _{x \in X}\left\|Q(x)-Q^{\prime}(x)\right\|+\max _{x \in X}\left(\left\|\Phi(x)-\Phi^{\prime}(x)\right\|+\left\|\partial_{1} \Phi(x)-\partial_{1} \Phi^{\prime}(x)\right\|\right. \\
& \left.\quad+\left\|\partial_{2} \Phi(x)-\partial_{2} \Phi^{\prime}(x)\right\|\right), \\
& d\left(B, B^{\prime}\right)=\left\|B-B^{\prime}\right\|_{C^{1}(\partial x)}=\max _{x \in \partial X}\left(\left\|B(x)-B^{\prime}(x)\right\|+\left\|\partial_{s} B(x)-\partial_{s} B^{\prime}(x)\right\|\right),
\end{aligned}
$$

where $s$ is the length parameter on $\partial X$. Here we use any of the standard norms on the spaces $B\left(\mathbb{C}^{N}\right)$ and $B\left(\mathbb{C}^{2 N}\right)$ of complex $N \times N$ and $2 N \times 2 N$ matrices, and on the space $\mathrm{B}\left(\mathbb{R}^{2}\right)$ of real $2 \times 2$ matrices.

Note that $\left(D_{\Phi_{t}, Q_{t}}, B_{t}\right)$ is a continuous path in $\mathcal{D} \times \mathcal{B}$ if and only if $Q_{t}(x), \Phi_{t}(x)$, $B_{t}(x)$, and the first partial derivatives of $\Phi_{t}(x), B_{t}(x)$ with respect to $x$ are continuous functions of $(t, x)$.

Denote by $\mathrm{HF}(\mathrm{H})$ the space of closed self-adjoint (or, what is the same, Hermitian) Fredholm operators on a separable Hilbert space $H$. We fix the graph topology on $\mathrm{HF}(\mathrm{H})$. Nevertheless we will usually write "graph continuous" instead of just "continuous" for the maps to $\mathrm{HF}(\mathrm{H})$ to avoid any misunderstanding.

By Lemma 1, we have the natural inclusion $\mathcal{D} \times \mathcal{B} \hookrightarrow \mathrm{HF}\left(\mathrm{L}^{2}\left(\mathrm{X}, \mathrm{g} ; \mathbb{C}^{2 \mathrm{~N}}\right)\right)$, which carries a pair $(\mathrm{D}, \mathrm{B}) \in \mathcal{D} \times \mathcal{B}$ to the operator $\mathrm{D}$ with the domain (18).

Lemma 2. The natural inclusion $\mathcal{D} \times \mathcal{B} \hookrightarrow \mathrm{HF}\left(\mathrm{L}^{2}\left(\mathrm{X}, \mathrm{g} ; \mathbb{C}^{2 \mathrm{~N}}\right)\right)$ is graph continuous.

Therefore, if $t \mapsto\left(D_{t}, B_{t}\right)$ is a continuous path in $\mathcal{D} \times \mathcal{B}$, then $\left(D_{t}, B_{t}\right)$ defines a graph continuous path in $\operatorname{HF}\left(\mathrm{L}^{2}\left(X, g ; \mathbb{C}^{2 N}\right)\right)$, and the spectral flow of the operator family $\left(D_{t}, B_{t}\right)$ is well defined.

Proof. Let us consider the smooth map

$\psi: \mathrm{B}\left(\mathbb{C}^{\mathrm{N}}\right) \rightarrow \mathrm{H}\left(\mathbb{C}^{2 \mathrm{~N}}\right), \quad \mathrm{A} \mapsto \mathrm{P}=\left(\begin{array}{cc}\mathrm{I}_{\mathrm{N}} & -\mathrm{A} \\ -A^{*} & A^{*} A\end{array}\right)\left(\begin{array}{cc}\mathrm{I}_{\mathrm{N}}+A A^{*} & 0 \\ 0 & \mathrm{I}_{N}+A^{*} A\end{array}\right)^{-1}$,

which carries $A \in B\left(\mathbb{C}^{N}\right)$ into the orthogonal projection $P$ of $\mathbb{C}^{2 N}$ with Ker $P=$ $\left\{u=\left(u^{+}, u^{-}\right): u^{+}, u^{-} \in \mathbb{C}^{N}, u^{+}=A u^{-}\right\}$. It induces the continuous map

$$
\psi_{*}: \mathrm{C}^{1}\left(\partial X, \mathrm{~B}\left(\mathbb{C}^{\mathrm{N}}\right)\right) \rightarrow \mathrm{C}^{1}\left(\partial X, \mathrm{H}\left(\mathbb{C}^{2 \mathrm{~N}}\right)\right) \text {. }
$$

Composing $\psi_{*}$ with the continuous map

$$
\mathcal{D} \times \mathcal{B} \rightarrow \mathrm{C}^{1}\left(\partial x, \mathrm{~B}\left(\mathbb{C}^{\mathrm{N}}\right)\right), \quad(\mathrm{D}, \mathrm{B}) \mapsto-i \rho^{+}(x, \mathrm{n}(x))^{-1} \mathrm{~B}(x),
$$


we obtain the continuous map

$$
\Psi: \mathcal{D} \times \mathcal{B} \rightarrow \mathrm{C}^{1}\left(\partial X, \mathrm{H}\left(\mathbb{C}^{2 \mathrm{~N}}\right)\right)
$$

which carries $(D, B)$ into the orthogonal projection $P$ of $L^{2}\left(\partial X,\left.g\right|_{\partial x} ; \mathbb{C}^{2 N}\right)$ with kernel defined by the boundary condition $(10) .^{3}$

By Proposition II.1.1 from [17], we have continuous inclusion of the Banach spaces

$$
\mathrm{B}\left(\mathrm{L}_{1}^{2}\left(\partial \mathrm{X} ; \mathbb{C}^{2 \mathrm{~N}}\right)\right) \subset \mathrm{B}\left(\mathrm{L}_{1 / 2}^{2}\left(\partial \mathrm{X} ; \mathbb{C}^{2 \mathrm{~N}}\right)\right),
$$

where $B(V)$ denotes the space of bounded linear operators on a Banach space $V, L_{r}^{2}$ is the (fractional) Sobolev space. Composing it with the natural continuous inclusion

$$
\mathrm{C}^{1}\left(\partial X, \mathrm{~B}\left(\mathbb{C}^{2 \mathrm{~N}}\right)\right) \subset \mathrm{B}\left(\mathrm{L}_{1}^{2}\left(\partial \mathrm{X} ; \mathbb{C}^{2 \mathrm{~N}}\right)\right),
$$

we obtain that the map $\Psi_{*}: \mathcal{D} \times \mathcal{B} \rightarrow \mathrm{B}\left(\mathrm{L}_{1 / 2}^{2}\left(\partial X ; \mathbb{C}^{2 \mathrm{~N}}\right)\right)$ is continuous.

The natural map from $\mathcal{D}$ to the space of bounded linear operators from $L_{1}^{2}\left(X ; \mathbb{C}^{2 N}\right)$ to $\mathrm{L}^{2}\left(\mathrm{X} ; \mathbb{C}^{2 \mathrm{~N}}\right)$ is continuous too:

$$
\begin{aligned}
\left\|\mathrm{D}_{\Phi, \mathrm{Q}}-\mathrm{D}_{\Phi^{\prime}, \mathrm{Q}^{\prime}}\right\|_{1,0} & \leqslant \mathrm{const}\left(\left\|\Phi-\Phi^{\prime}\right\|_{\mathrm{C}(\mathrm{X})}+\left\|\mathrm{R}_{\Phi}-\mathrm{R}_{\Phi^{\prime}}\right\|_{\mathrm{C}(\mathrm{X})}+\left\|\mathrm{Q}-\mathrm{Q}^{\prime}\right\|_{\mathrm{C}(\mathrm{X})}\right) \\
& \leqslant \mathrm{const}\left(\left\|\Phi-\Phi^{\prime}\right\|_{\mathrm{C}^{1}(\mathrm{X})}+\left\|\mathrm{Q}-\mathrm{Q}^{\prime}\right\|_{\mathrm{C}(\mathrm{X})}\right) .
\end{aligned}
$$

By Theorem 7.16 from [7] and by Lemma 1, this implies that the inclusion $\mathcal{D} \times \mathcal{B} \hookrightarrow$ $\mathrm{HF}\left(\mathrm{L}^{2}\left(\mathrm{X}, \mathrm{g} ; \mathbb{C}^{2}\right)\right)$ is graph continuous. This completes the proof.

\section{Basic Properties of the Spectral Flow}

There can be different versions of the definition of the spectral flow when one or both of the endpoints of the path is non-invertible. If a path is a loop up to a gauge transformation as in the first part of the paper, then the value of the spectral flow is independent of the choice of definition. But for the proofs below we have to fix some choice.

Definition. Let $\left(A_{t}\right)$ be a 1-parameter graph continuous family of closed self-adjoint Fredholm operators on a separable complex Hilbert space $\mathrm{H}$. Take a small $\varepsilon>0$ such that $A_{0}, A_{1}$ have no eigenvalues in the interval $[-\varepsilon, 0)$. We put $\mathrm{sf}\left(A_{t}\right)$ equal to $\operatorname{sf}\left(A_{t}+\varepsilon I\right)$, where we use any of the (equivalent) definitions of the spectral flow for the path of self-adjoint Fredholm operators with invertible endpoints from [6,12]. This definition does not depend on the choice of $\varepsilon$.

We will need the following properties of the spectral flow.

(P0) Zero crossing. In the absence of zero crossing the spectral flow vanishes. More precisely, suppose $\gamma:[0,1] \rightarrow \mathcal{D} \times \mathcal{B}$ is the continuous path such that 0 is not an eigenvalue of $\gamma(\mathrm{t})$ for any $\mathrm{t} \in[0,1]$. Then $\mathrm{sf}(\gamma)=0$.

(P1) Homotopy invariance. The spectral flow along the continuous path $\gamma:[0,1] \rightarrow$ $\mathcal{D} \times \mathcal{B}$ does not change when $\gamma$ changes continuously in the space of paths in $\mathcal{D} \times \mathcal{B}$ with fixed endpoints (the same as the endpoints of $\gamma$ ).

\footnotetext{
${ }^{3}$ Here we use the general formula for the orthogonal projection $\mathrm{P}$ with the kernel $\left\{\mathrm{u}^{+}=\mathrm{A} u^{-}\right\}$for arbitrary matrix $A$. Actually, in our case $A=\left(\mathrm{in}^{\prime}\right)^{-1} B$ is normal: $A A^{*}=A^{*} A$.
} 
In other words, for the continuous map $\mathrm{h}:[0,1] \times[0,1] \rightarrow \mathcal{D} \times \mathcal{B}$ such that $\mathrm{h}_{\mathrm{s}}(0) \equiv$ $\left(D_{0}, B_{0}\right), h_{s}(1) \equiv\left(D_{1}, B_{1}\right)$, we have $s f\left(h_{0}(t)\right)_{t \in[0,1]}=s f\left(h_{1}(t)\right)_{t \in[0,1]}$.

(P2) Path additivity. Suppose $\gamma:[\mathrm{a}, \mathrm{c}] \rightarrow \mathcal{D} \times \mathcal{B}$ is a continuous path, $\mathrm{a} \leqslant \mathrm{b} \leqslant \mathrm{c}$. Then $\mathrm{sf}(\gamma(\mathrm{t}))_{\mathrm{t} \in[\mathrm{a}, \mathrm{c}]}=\mathrm{sf}(\gamma(\mathrm{t}))_{\mathrm{t} \in[\mathrm{a}, \mathrm{b}]}+\mathrm{sf}(\gamma(\mathrm{t}))_{\mathrm{t} \in[\mathrm{b}, \mathrm{c}]}$.

(P3) Additivity with respect to direct sum. Let $\mathrm{N}_{1}, \mathrm{~N}_{2}$ be natural numbers, $\left(\mathrm{D}_{\mathrm{t}}^{\mathrm{i}}, \mathrm{B}_{\mathrm{t}}^{\mathrm{i}}\right)$ be continuous paths in $\mathcal{D}_{\mathrm{N}_{i}} \times \mathcal{B}_{\mathrm{N}_{i}}$. Then the spectral flow along the path $\left(\mathrm{D}_{\mathrm{t}}^{1} \oplus \mathrm{D}_{\mathrm{t}}^{2}, \mathrm{~B}_{\mathrm{t}}^{1} \oplus \mathrm{B}_{\mathrm{t}}^{2}\right)$ is equal to the sum of the spectral flows along the paths $\left(\mathrm{D}_{\mathrm{t}}^{1}, \mathrm{~B}_{\mathrm{t}}^{1}\right)$ and $\left(\mathrm{D}_{\mathrm{t}}^{2}, \mathrm{~B}_{\mathrm{t}}^{2}\right)$.

(P4) Conjugacy invariance. Let $\mathrm{J}_{ \pm}: \mathrm{X} \rightarrow \mathrm{U}(\mathrm{N})$ be unitary $\mathrm{N} \times \mathrm{N}$ matrices smoothly dependent on $\mathrm{x} \in \mathrm{X}$, set $\mathrm{J}=\left(\begin{array}{cc}\mathrm{J}_{+} & 0 \\ 0 & \mathrm{~J}_{-}\end{array}\right): \mathrm{X} \rightarrow \mathrm{U}(2 \mathrm{~N})$, and let $\left(\mathrm{D}_{\mathrm{t}}, \mathrm{B}_{\mathrm{t}}\right)$ be a smooth path in $\mathcal{D} \times \mathcal{B}$. Then $\mathrm{sf}\left(\mathrm{D}_{\mathrm{t}}, \mathrm{B}_{\mathrm{t}}\right)=\mathrm{sf}\left(\mathrm{JD}_{\mathrm{t}} \mathrm{J}^{-1}, \mathrm{~J}_{-} \mathrm{B}_{\mathrm{t}} \mathrm{J}_{-}^{-1}\right)$.

More generally, if $\mathrm{H}$ is a separable complex Hilbert space, J is an unitary isomorphism of $\mathrm{H},\left(A_{\mathrm{t}}\right)$ is a 1-parameter graph continuous family of closed self-adjoint Fredholm operators, then $\operatorname{sf}\left(A_{t}\right)=\operatorname{sf}\left(J A_{t} J^{-1}\right)$.

Remark. Properties (P1) and (P2) imply that the spectral flow along the path is opposite to the spectral flow along the same path passing in the opposite direction.

Proof. By Lemmas 1-2, the inclusion of $\mathcal{D} \times \mathcal{B}$ into HF $\left(\mathrm{L}^{2}\left(\mathrm{X}, \mathrm{g} ; \mathbb{C}^{2 \mathrm{~N}}\right)\right)$ is graph continuous. So it is sufficient to prove Properties (P0-P4) for graph continuous paths in the space $\mathrm{HF}(\mathrm{H})$ of all closed self-adjoint Fredholm operators in a separable Hilbert space $\mathrm{H}$; this will imply Properties (P0-P4) for the paths in $\mathcal{D} \times \mathcal{B}$.

The first three properties of the spectral flow for graph continuous paths in $\operatorname{HF}(H)$ are proved in [6] (Prop. 2.2), taking into account the convention from Sect. 2 for the case when $\gamma(0)$ or $\gamma(1)$ are non-invertible.

Properties (P3-P4) for graph continuous paths in $\mathrm{HF}(\mathrm{H})$ are obviously fulfilled for the definition of spectral flow via spectral projections ([6], Def. 2.12). For the definition of spectral flow via the Cayley transform ([6], Def. 2.2) they can be proved easily using the uniqueness property of the spectral flow; we give here these proofs for completeness (though the two definitions of spectral flow are equivalent [6], the proof of their equivalence is not very simple).

To prove ( $\mathrm{P} 4)$, let us consider a unitary isomorphism $J$ of a separable complex Hilbert space $H$. To each graph continuous path $\left(A_{t}\right)$ in $\operatorname{HF}(H)$ assign the integer $\operatorname{sf}_{\text {new }}\left(A_{t}\right)=$ $\operatorname{sf}\left(\mathrm{JA}_{\mathrm{t}} \mathrm{J}^{-1}\right)$. Then $\mathrm{sf}_{\text {new }}$ satisfies Concatenation, Homotopy and Normalization properties in the sense of [12]. By Theorem 5.9 from [12], this implies that $s f_{n e w}$ equals sf for the paths in $\operatorname{HF}(H)$ with invertible endpoints. Taking into account our convention from Sect. 2 and choosing a small $\varepsilon>0$ such that $A_{0}, A_{1}$ have no eigenvalues in the interval $[-\varepsilon, 0)$, we obtain

$$
\operatorname{sf}\left(J A_{t} J^{-1}\right)=\operatorname{sf}\left(J A_{t} J^{-1}+\varepsilon I\right)=\operatorname{sf}_{\text {new }}\left(A_{t}+\varepsilon I\right)=\operatorname{sf}\left(A_{t}+\varepsilon I\right)=\operatorname{sf}\left(A_{t}\right) .
$$

To prove (P3), consider graph continuous paths $\left(A_{t}\right),\left(A_{t}^{\prime}\right)$ in $\operatorname{HF}(H), H F\left(H^{\prime}\right)$ respectively. Suppose for a while that $A_{0}, A_{1}, A_{0}^{\prime}$, and $A_{1}^{\prime}$ are invertible. The path $\left(A_{t} \oplus A_{t}^{\prime}\right)_{t \in[0,1]}$ is homotopic to the concatenation of paths $\left(A_{t} \oplus A_{0}^{\prime}\right)_{t \in[0,1]}$ and $\left(A_{1} \oplus A_{t}^{\prime}\right)_{t \in[0,1]}$ in $\mathrm{HF}\left(H \oplus \mathrm{H}^{\prime}\right)$. The spectral flow of the path $\left(A_{t} \oplus A_{0}^{\prime}\right)$ in $\mathrm{HF}\left(H \oplus H^{\prime}\right)$ considered as a function of $\left(A_{t}\right)$ satisfies Concatenation, Homotopy and Normalization properties in the sense of [12], so by the uniqueness property of the spectral 
flow from [12] we have sf $\left(A_{t} \oplus A_{0}^{\prime}\right)=\operatorname{sf}\left(A_{t}\right)$. Similarly, sf $\left(A_{1} \oplus A_{t}^{\prime}\right)=\operatorname{sf}\left(A_{t}^{\prime}\right)$. Therefore, sf $\left(A_{t} \oplus A_{t}^{\prime}\right)=\operatorname{sf}\left(A_{t}\right)+\operatorname{sf}\left(A_{t}^{\prime}\right)$ for any paths $\left(A_{t}\right),\left(A_{t}^{\prime}\right)$ with invertible endpoints. Taking into account our convention from Sect. 2, we obtain that sf $\left(A_{t} \oplus A_{t}^{\prime}\right)=\operatorname{sf}\left(A_{t}\right)+\operatorname{sf}\left(A_{t}^{\prime}\right)$ for arbitrary paths $\left(A_{t}\right),\left(A_{t}^{\prime}\right)$. This completes the proof.

\section{Independence of the Choice of the Family $\left(Q_{t}\right)$}

Let us prove that the spectral flow along $\left(D+Q_{t}, B\right)$ does not depend on the choice of $\left(Q_{t}\right)$ when $D, B, \mu$ are fixed.

Let $Q_{t}, Q_{t}^{\prime}$ be two 1-parameter families of smooth maps from $X$ to $H\left(\mathbb{C}^{2 N}\right)$ such that $\mathrm{Q}_{0}=\mathrm{Q}_{0}^{\prime}=0, \mathrm{Q}_{1}=\mathrm{Q}_{1}^{\prime}=\mu \mathrm{D} \mu^{-1}-\mathrm{D}$.

The path $D+Q_{t}$ can be continuously changed to the path $D+Q_{t}^{\prime}$ in the class of paths in $\mathcal{D}$ with the fixed endpoints. For example, we can take the homotopy $h(s, t)=$ $\mathrm{D}+(1-\mathrm{s}) \mathrm{Q}_{\mathrm{t}}+\mathrm{s} \mathrm{Q}_{\mathrm{t}}^{\prime}$. By the homotopy invariance property (P1) of the spectral flow, sf $\left(D+Q_{t}, B\right)_{t \in[0,1]}=s f\left(D+Q_{t}^{\prime}, B\right)_{t \in[0,1]}$.

Therefore, if $\mathrm{Q}_{0}=0, \mathrm{Q}_{1}=\mu \mathrm{D} \mu^{-1}-\mathrm{D}$ then

$$
\text { sf }\left(D+Q_{t}, B\right)_{t \in[0,1]}=F(X, g, N, D, B, \mu)
$$

for some integer-valued function F. Now we will investigate the properties of this function.

\section{Independence of the Choice of Operator D}

1. Suppose that $D_{0}$ is homotopic to $D_{1}$ in $\mathcal{D}$, that is there exist a continuous 1-parameter family of Dirac type operators $D_{s}$ connecting $D_{0}$ with $D_{1}$. We will show that $F\left(X, g, N, D_{0}, B, \mu\right)=F\left(X, g, N, D_{1}, B, \mu\right)$.

Let us consider the 2-parameter family of Dirac type operators $D_{s, t}=(1-t) D_{s}+$ $t \mu D_{s} \mu^{-1}$. Note that $D_{s, 0}=D_{s}, D_{s, 1}=\mu D_{s} \mu^{-1}, D_{s, t}-D_{s, 0}=t Q_{s}$, where $Q_{s}=\mu D_{s} \mu^{-1}-D_{s}$ is a 1-parameter family of $2 \mathrm{~N} \times 2 \mathrm{~N}$ self-adjoint complex matrices smoothly dependent on $x \in X$.

Let us define the path $\gamma_{1}:[0,3] \rightarrow \mathcal{D}$ by the formula

$$
\gamma_{1}(t)= \begin{cases}D_{t, 0}, & t \in[0,1] \\ D_{1, t-1}, & t \in[1,2] . \\ D_{3-t, 1}, & t \in[2,3]\end{cases}
$$

In other words, we sequentially go around the left, top and right sides of the rectangle on Fig. 4 in a clockwise direction. The path $\gamma_{1}$ can be continuously deformed to the path

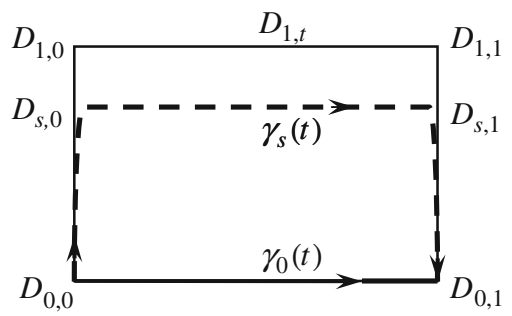

Fig. 4. Homotopy from $\gamma_{0}(t)$ to $\gamma_{1}(t)$ 
$\mathrm{D}_{0, \mathrm{t}}=\mathrm{D}_{0}+\mathrm{t} \mathrm{Q}_{0}$ within the rectangle. For example, we can take as such a deformation the family

$$
\gamma_{s}(t)=\left\{\begin{array}{ll}
D_{s t, 0}, & t \in[0,1] \\
D_{s, t-1}, & t \in[1,2] \\
D_{s(3-t), 1}, & t \in[2,3]
\end{array} .\right.
$$

Then $\gamma_{0}(t)$ is the path $\left(D_{0}+(t-1) Q_{0}\right)_{t \in[1,2]}$ concatenated with two constant paths, the spectral flows along which are zero by Property (P0).

By the homotopic invariance property of the spectral flow,

$$
\operatorname{sf}\left(\gamma_{1}(t), B\right)_{t \in[0,3]}=s f\left(\gamma_{0}(t), B\right)_{t \in[0,3]}=s f\left(D_{0}+t Q_{0}, B\right)_{t \in[0,1]} .
$$

On the other hand, the spectral flows along the first and the third parts of $\gamma_{1}$ are mutually reduced by $(\mathrm{P} 4)$ :

$$
\begin{aligned}
& \operatorname{sf}(\gamma(t), B)_{t \in[0,1]}+\operatorname{sf}(\gamma(t), B)_{t \in[2,3]} \\
& \quad=s f\left(D_{s}, B\right)_{s \in[0,1]}-s f\left(\mu D_{s} \mu^{-1}, B\right)_{s \in[0,1]}=0 .
\end{aligned}
$$

Therefore,

$$
s f\left(D_{0}+t Q_{0}, B\right)_{t \in[0,1]}=s f\left(\gamma_{1}(t), B\right)_{t \in[1,2]}=s f\left(D_{1}+t Q_{1}, B\right)_{t \in[0,1]},
$$

and $F\left(X, g, N, D_{0}, B, \mu\right)=F\left(X, g, N, D_{1}, B, \mu\right)$.

2. Now we will simplify D step by step.

At first, we can continuously change $D=D_{\Phi, Q}=-i\left(\rho_{1} \partial_{1}+\rho_{2} \partial_{2}\right)+i R_{\Phi}(x)+$ $\mathrm{Q}(x)$ to the operator $\mathrm{D}_{\Phi, 0}$, for example, along the path $\mathrm{D}_{\Phi,(1-\mathrm{s}) \mathrm{Q}}$.

Further, take a smooth map $h:[0,1] \times \mathrm{GL}^{+}(2, \mathbb{R}) \rightarrow \mathrm{GL}^{+}(2, \mathbb{R})$ such that $h(0, \cdot)$ is the identity map, while the image of $h(1, \cdot)$ is the group $S O(2, \mathbb{R})$ of $2 \times 2$ orthogonal real matrices with determinant equal to one (the existence of such a family is well known in any dimension; this is an easy application of the Gram-Schmidt orthonormalisation procedure). The operator $\mathrm{D}_{\Phi, 0}$ can be continuously changed in $\mathcal{D}$ along the path $\mathrm{D}_{\mathrm{h}(\mathrm{t}, \Phi), 0}$ to the operator $\mathrm{D}_{\Phi^{\prime}, 0}$, where

$$
\Phi^{\prime}(x)=\left(\begin{array}{cc}
\cos \varphi & \sin \varphi \\
-\sin \varphi & \cos \varphi
\end{array}\right) \in \operatorname{SO}(2, \mathbb{R})
$$

for some smooth function $\varphi$ from $X$ to $S^{1}$. So we have

$$
\mathrm{F}\left(\mathrm{X}, \mathrm{g}, \mathrm{N}, \mathrm{D}_{\Phi, \mathrm{Q}}, \mathrm{B}, \mu\right)=\mathrm{F}\left(\mathrm{X}, \mathrm{g}, \mathrm{N}, \mathrm{D}_{\Phi^{\prime}, 0}, \mathrm{~B}, \mu\right) .
$$

On the other hand, $\mathrm{D}_{\Phi^{\prime}, 0}$ can be represented as $\mathrm{J}^{-1} \mathrm{D}_{\mathrm{I}, 0} \mathrm{~J}$, where $\mathrm{I}=\mathrm{I}_{2}$ is the identity $2 \times 2$ matrix and

$$
J(x)=\left(\begin{array}{cc}
J_{+} & 0 \\
0 & J_{-}
\end{array}\right)=\left(\begin{array}{cc}
e^{i \varphi} I_{N} & 0 \\
0 & I_{N}
\end{array}\right) \in U(2 N) .
$$

Let $\mathrm{Q}_{t}$ be a 1-parameter family of self-adjoint $2 \mathrm{~N} \times 2 \mathrm{~N}$ complex matrices such that $\mathrm{Q}_{0}=0, \mathrm{Q}_{1}=\mu \mathrm{D}_{\Phi^{\prime}, 0} \mu^{-1}-\mathrm{D}_{\Phi^{\prime}, 0}$. Applying Property (P4) of the spectral flow, we obtain

$$
\begin{aligned}
\mathrm{F}\left(X, \mathrm{~g}, \mathrm{~N}, \mathrm{D}_{\Phi^{\prime}, 0}, \mathrm{~B}, \mu\right) & =\operatorname{sf}\left(\mathrm{D}_{\Phi^{\prime}, 0}+\mathrm{Q}_{\mathrm{t}}, \mathrm{B}\right)=\operatorname{sf}\left(\mathrm{J}_{\left.\left(\Phi_{\Phi^{\prime}, 0}+\mathrm{Q}_{\mathrm{t}}\right) \mathrm{J}^{-1}, \mathrm{~J}_{-} \mathrm{BJ}_{-}^{-1}\right)}\right. \\
& =\operatorname{sf}\left(\mathrm{D}_{\mathrm{I}, 0}+\mathrm{JQ}_{\mathrm{t}} \mathrm{J}^{-1}, \mathrm{~B}\right)=\mathrm{F}\left(X, \mathrm{~g}, \mathrm{~N}, \mathrm{D}_{\mathrm{I}, 0}, \mathrm{~B}, \mu\right)
\end{aligned}
$$


because

$$
\mathrm{JQ}_{1} \mathrm{~J}^{-1}=\mu\left(\mathrm{JD}_{\Phi^{\prime}, 0} \mathrm{~J}^{-1}\right) \mu^{-1}-\mathrm{JD}_{\Phi^{\prime}, 0} \mathrm{~J}^{-1}=\mu \mathrm{D}_{\mathrm{I}, 0} \mu^{-1}-\mathrm{D}_{\mathrm{I}, 0} .
$$

Therefore, $F(X, g, N, D, B, \mu)$ does not depend on the choice of $D \in \mathcal{D}$, so from now on we will write $F(X, g, N, B, \mu)$ instead of $F(X, g, N, D, B, \mu)$.

\section{Independence of the Metric and Invariance Under a Change of Variables}

We prove here that $F(X, g, N, B, \mu)$ is independent of the choice of metric $g$ on $X$, invariant under the change of variables, and does not depend on the geometry of $X$, using the fact that the number of holes is the only topological invariant of a disk with holes, and that the spectral flow is conjugacy invariant and does not depend on the choice of the operator.

Let $X, X^{\prime}$ be compact planar domains, each bounded by $m$ smooth curves, and let $g$, $g^{\prime}$ be Riemannian metrics on $X, X^{\prime}$ respectively.

As is well known, there exists an orientation-preserving diffeomorphism $f: X^{\prime} \rightarrow X{ }^{4}$

We define $\theta$ as the smooth function from $X^{\prime}$ to $\mathbb{R}^{+}$such that $f^{*} \mathrm{dvol}=\theta \mathrm{dvol}^{\prime}$, where dvol, $\mathrm{dvol}^{\prime}$ are volume elements on $X, X^{\prime}$ respectively. ${ }^{5}$

The diffeomorphism $f$ defines a unitary isomorphism $J$ from the Hilbert space $\mathrm{L}^{2}\left(X, g ; \mathbb{C}^{2 \mathrm{~N}}\right)$ to the Hilbert space $\mathrm{L}^{2}\left(\mathrm{X}^{\prime}, \mathrm{g}^{\prime} ; \mathbb{C}^{2 \mathrm{~N}}\right), \mathrm{u} \mapsto \sqrt{\theta} f^{*} u .^{6}$

The isomorphism $J$ transforms the operator $\mathrm{D} \in \mathcal{D}_{X, g, N}$ with symbol $\rho$ to a symmetric operator $D^{\prime}=J_{D J}^{-1}$ on $X^{\prime}$ with symbol $\rho^{\prime}$. For any $x^{\prime} \in X^{\prime}, x=f\left(x^{\prime}\right)$, any cotangent vector $\xi \in T_{x}^{*} X, \xi^{\prime}=f^{*} \xi$, we have $\rho^{\prime}\left(x^{\prime}, \xi^{\prime}\right)=\rho(x, \xi)$, that is $\rho^{\prime}\left(x^{\prime}\right)=$ $\left(\frac{\partial x^{\prime}}{\partial x}\right) \rho(x)=\left(\frac{\partial x^{\prime}}{\partial x}\right) \Phi \sigma$ in coordinate representation. The matrix $\left(\frac{\partial x^{\prime}}{\partial x}\right) \Phi(x)$ is contained in $\mathrm{GL}^{+}(2, \mathbb{R})$ for any $x \in X$, so $\mathrm{D}^{\prime} \in \mathcal{D}_{\mathrm{X}^{\prime}, \mathrm{g}^{\prime}, \mathrm{N}}$.

Let $\mu$ be a smooth function from $X$ to $U(1)$. Taking the map $\mu^{\prime}=f^{*} \mu$ from $X^{\prime}$ to $\mathrm{U}(1)$ and the map $B^{\prime}=\left\|f^{*} n\right\|_{g^{\prime}}^{-1} f^{*} B$ from $\partial X^{\prime}$ to $H\left(\mathbb{C}^{N}\right)$, we obtain

$$
\mu^{\prime} \mathrm{D}^{\prime} \mu^{\prime-1}-\mathrm{D}^{\prime}=\mu^{\prime}\left(\mathrm{JDJ}^{-1}\right) \mu^{\prime-1}-\mathrm{JDJ}^{-1}=\mathrm{J}\left(\mu \mathrm{D} \mu^{-1}-\mathrm{D}\right) \mathrm{J}^{-1} \text {. }
$$

So if $\mathrm{Q}_{\mathrm{t}}$ connects $\mathrm{Q}_{0}=0$ with $\mathrm{Q}_{1}=\mu \mathrm{D} \mu^{-1}-\mathrm{D}$, then $\mathrm{Q}_{\mathrm{t}}^{\prime}=\mathrm{JQ}_{\mathrm{t}} \mathrm{J}^{-1}$ connects $\mathrm{Q}_{0}^{\prime}=0$ with $\mathrm{Q}_{1}^{\prime}=\mu^{\prime} \mathrm{D}^{\prime} \mu^{\prime-1}-\mathrm{D}^{\prime}$, and by the conjugacy invariance of the spectral flow (P4), we have

$$
\operatorname{sf}\left(D+Q_{t}, B\right)=s f\left(J\left(D+Q_{t}\right) J^{-1}, B^{\prime}\right)=s f\left(D^{\prime}+Q_{t}^{\prime}, B^{\prime}\right) .
$$

However, $B^{\prime}$ is homotopic to $f^{*} B$ in $\mathcal{B}_{X^{\prime}, N}$, while the spectral flow of $\left(D^{\prime}+Q_{t}^{\prime}, \widetilde{B}\right)$ is invariant under the continuous change of $\widetilde{B}$ in $\mathcal{B}_{X^{\prime}, N}$ (this is verified in a way similar to

\footnotetext{
4 In other words, there exists a smooth one-to-one change of variables $\left(x^{\prime 1}, x^{\prime 2}\right)=x^{\prime} \stackrel{f}{\rightarrow} x=\left(x^{1}, x^{2}\right)$ with the smooth inverse and with positive Jacobian determinant $\operatorname{det}\left(\partial x / \partial x^{\prime}\right)$, which transforms $X^{\prime}$ onto $X$.

5 As usual, by $f^{*}$ we denote the homomorphism from differential forms (in particular, functions) on $X$ to differential forms on $X^{\prime}$, which is induced by $f$. In coordinate form, $\theta\left(x^{\prime}\right)=\frac{\sqrt{g\left(f\left(x^{\prime}\right)\right)}}{\sqrt{g^{\prime}\left(x^{\prime}\right)}} \operatorname{det}\left(\frac{\partial x}{\partial x^{\prime}}\right)$.

6 That is $(J u)\left(x^{\prime}\right)=\sqrt{\theta\left(x^{\prime}\right)} u\left(f\left(x^{\prime}\right)\right)$.
} 
the proof in Sect. 13). Therefore $s f\left(D^{\prime}+Q_{t}^{\prime}, B^{\prime}\right)=s f\left(D^{\prime}+Q_{t}^{\prime}, f^{*} B\right)$, and finally we obtain

$$
F(X, g, N, B, \mu)=F\left(X^{\prime}, g^{\prime}, N, f^{*} B, f^{*} \mu\right)
$$

This completes the proof.

In particular, for any two metrics $\mathrm{g}, \mathrm{g}^{\prime}$ on the same $\mathrm{X}$, using the identity diffeomorphism $f$, we have

$$
F(X, g, N, B, \mu)=F\left(X, g^{\prime}, N, B, \mu\right)
$$

From now on we will write $F(N, B, \mu)$ instead of $F(X, g, N, B, \mu)$.

\section{Boundary Conditions}

Let us investigate the dependence of $F(N, B, \mu)$ on $B$.

$F(N, B, \mu)$ does not change when $B$ changes continuously in $\mathcal{B}$; this is verified in a way similar to the proof in Sect. 13.

Let $b_{j}$ be the number of negative eigenvalues of $B$ (counting multiplicities) on $\partial X_{j}$. We prove that the ordered set $\hat{b}=\left(b_{j}\right)_{j=1}^{m}$ uniquely determines $B$ up to continuous variation of $B$ in $\mathcal{B}$.

Obviously, $\hat{b}$ is invariant with respect to such variations, so we only have to prove that any two $B, B^{\prime}$ with the same $\hat{b}$ are homotopic. It is sufficient to prove that any smooth map $A$ from the circle $S^{1}$ to the space of complex self-adjoint invertible $\mathrm{N} \times \mathrm{N}$ matrices is homotopic (in the space of all such maps with $C^{1}$-metric) to the constant map sending $S^{1}$ to the point $\left(-I_{k}\right) \oplus I_{N-k} \in H\left(\mathbb{C}^{N}\right)$, where $k$ is the number of negative eigenvalues of $A(x), x \in S^{1}$.

1. Let us consider the continuous 1-parameter family $A_{S}$ of smooth maps from $S^{1}$ to the space of complex self-adjoint invertible $\mathrm{N} \times \mathrm{N}$ matrices defined by the formula $A_{s}=A \cdot\left((1-s) I_{N}+s A^{2}\right)^{-1 / 2}$. This expression is correct because $(1-s) I_{N}+s A^{2}$ is self-adjoint and positive definite for any $s \in[0,1]$. The family $A_{s}$ gives us a deformation from $A=A_{0}$ to a smooth map $A_{1}$ from $S^{1}$ to the space of self-adjoint unitary $\mathrm{N} \times \mathrm{N}$ matrices.

2. The connected component of $A_{1}(x)$ in the space of self-adjoint unitary $N \times N$ matrices is diffeomorphic to the space $\mathrm{Gr}_{\mathbb{C}}(k, N)$ of all $k$-dimensional linear subspaces of $\mathbb{C}^{\mathrm{N}}$. This diffeomorphism is defined by the correspondence $\mathrm{U} \mapsto \operatorname{Ker}\left(\mathrm{I}_{\mathrm{N}}+\mathrm{U}\right)$, which associates with $\mathrm{U}$ the invariant subspace $\mathrm{V} \subseteq \mathbb{C}^{\mathrm{N}}$ of $\mathrm{U}$ corresponding to eigenvalue -1 of $\mathrm{U}$. The inverse diffeomorphism is defined by the formula $\mathrm{V} \mapsto \mathrm{U}=(-\mathrm{I})_{\mathrm{V}} \oplus \mathrm{I}_{\mathrm{V} \perp}$.

The complex Grassmannian $\mathrm{Gr}_{\mathbb{C}}(k, N)$ is known to be simply connected, so any two continuous maps from the circle to $\mathrm{Gr}_{\mathbb{C}}(k, N)$ are homotopic. Taking into account that $\mathrm{Gr}_{\mathbb{C}}(k, N)$ is a smooth manifold, we obtain that the space of smooth maps from the circle to $\mathrm{Gr}_{\mathbb{C}}\left(\mathrm{k}, \mathrm{N}\right.$ ) (with $\mathrm{C}^{1}$-metric) is path-connected. The same is true for the connected component of the space of self-adjoint unitary $\mathrm{N} \times \mathrm{N}$ matrices which is diffeomorphic to $\mathrm{Gr}_{\mathbb{C}}(\mathrm{k}, \mathrm{N})$, so $A$ can be continuously changed in the class of smooth maps to the constant map $x \mapsto\left(-\mathrm{I}_{\mathrm{k}}\right) \oplus \mathrm{I}_{\mathrm{N}-\mathrm{k}}$. This completes the proof. 


\section{Gauge Transformations}

1. We will prove that $F$ is linear in $\mu$, that is $F\left(N, B, \mu_{1} \mu_{2}\right)=F\left(N, B, \mu_{1}\right)+F\left(N, B, \mu_{2}\right)$ for any smooth functions $\mu_{1}, \mu_{2}: X \rightarrow U(1)$.

Let $Q_{i}=\mu_{i} D \mu_{i}^{-1}-D$. Then $Q_{1}+Q_{2}=\left(\mu_{1} \mu_{2}\right) D\left(\mu_{1} \mu_{2}\right)^{-1}-D$, so by definition $\mathrm{F}\left(\mathrm{N}, \mathrm{B}, \mu_{1} \mu_{2}\right)$ is equal to the spectral flow along the path $\left(\mathrm{D}+\mathrm{P}_{\mathrm{t}}, \mathrm{B}\right)_{t \in[0,2]}$, where $P_{0}=0, P_{2}=Q_{1}+Q_{2}$. We can take $P_{t}$ composed from two parts: from 0 to $Q_{1}$ and then from $\mathrm{Q}_{1}$ to $\mathrm{Q}_{1}+\mathrm{Q}_{2}$, for example,

$$
P_{t}=\left\{\begin{array}{ll}
t Q_{1}, & t \in[0,1] \\
Q_{1}+(t-1) Q_{2}, & t \in[1,2]
\end{array} .\right.
$$

Using Property (P2) of the spectral flow, we obtain

$$
\begin{aligned}
\mathrm{F}\left(\mathrm{N}, \mathrm{B}, \mu_{1} \mu_{2}\right) & =\operatorname{sf}\left(\mathrm{D}+\mathrm{P}_{\mathrm{t}}, \mathrm{B}\right)_{\mathrm{t} \in[0,1]}+\operatorname{sf}\left(\mathrm{D}+\mathrm{P}_{\mathrm{t}}, \mathrm{B}\right)_{\mathrm{t} \in[1,2]} \\
& =\operatorname{sf}\left(\mathrm{D}+\mathrm{tQ} \mathrm{Q}_{1}, \mathrm{~B}\right)_{\mathrm{t} \in[0,1]}+\operatorname{sf}\left(\left(\mathrm{D}+\mathrm{Q}_{1}\right)+\mathrm{tQ}_{2}, \mathrm{~B}\right)_{\mathrm{t} \in[0,1]} \\
& =\mathrm{F}\left(\mathrm{N}, \mathrm{B}, \mu_{1}\right)+\mathrm{F}\left(\mathrm{N}, \mathrm{B}, \mu_{2}\right)
\end{aligned}
$$

so $\mathrm{F}$ is linear in $\mu$.

2. Denote by $M$ the set of equivalence classes of smooth functions $\mu: X \rightarrow U(1)$, where two functions are equivalent if one of them can be continuously changed to the other in the space of smooth functions from $X$ to $U(1)$ (with $C^{1}$-metric). We will consider $M$ as an Abelian group, where the group structure on $M$ is induced by the group structure on $\mathrm{U}(1)$. It is well known that

$$
M=\left\{\left(\mu_{1}, . ., \mu_{m}\right) \in \mathbb{Z}^{m}: \sum \mu_{j}=0\right\}
$$

with the group structure induced from $\mathbb{Z}^{m}$, and the class of $\mu$ in $M$ is defined by the m-tuple $\hat{\mu}=\left(\mu_{j}\right)$, where $\mu_{j}$ is the degree of the restriction of $\mu$ to $\partial X_{j}$.

Let us prove that $F(N, B, \mu)$ depends only on the class of $\mu$ in $M$.

Suppose that $\mu_{t}$ is a continuous path in the space of smooth functions from $X$ to $U(1)$ such that $\mu_{0}(x) \equiv 1$. By Part 1 of this section, it is sufficient to prove that $F\left(N, B, \mu_{1}\right)=0$. Let us take $Q_{t}=\mu_{t} D \mu_{t}{ }^{-1}-D$. Taking into account that $Q_{1}=\mu_{1} D \mu_{1}^{-1}-D$, we obtain $F\left(N, B, \mu_{1}\right)=s f\left(D+Q_{t}, B\right)$. But all the operators $\left(D+Q_{t}, B\right)$ are conjugate to $(D, B)$ by $\mu_{t}$ and therefore are isospectral. Let $\varepsilon>0$ be such that $(D, B)$ has no zero eigenvalues in the interval $[-\varepsilon, 0)$. Then $s f\left(D+Q_{t}, B\right)=s f\left(D+Q_{t}+\varepsilon I_{2 N}, B\right)=0$ by (P0) because all the operators $\left(D+Q_{t}+\varepsilon I_{2 N}, B\right)$ have no zero eigenvalues. This completes the proof.

\section{Bilinearity}

In the previous sections we have proven that $\mathrm{F}$ depends only on the integer numbers $\mathrm{N}$, $b_{1}, \ldots, b_{m}, \mu_{1}, \ldots, \mu_{m}$. Now we will study this dependence more closely.

By $S$ denote the set of all possible $(m+1)$-tuples $\left(N, b_{1}, \ldots, b_{m}\right)$ :

$$
S=\left\{\left(N, b_{1}, \ldots, b_{m}\right) \in \mathbb{Z}^{m+1}: N \geqslant 1,0 \leqslant b_{j} \leqslant N\right\}
$$


$F$ defines the map from $S \times M$ to $\mathbb{Z}$ (which we denote by the same letter $F$ for simplicity) satisfying the following conditions:

$$
\begin{aligned}
F\left(N, \hat{b}, \hat{\mu} \oplus \hat{\mu}^{\prime}\right) & =F(N, \hat{b}, \hat{\mu})+F\left(N, \hat{b}, \hat{\mu}^{\prime}\right), \\
F\left(N+N^{\prime}, \hat{b} \oplus \hat{b}^{\prime}, \hat{\mu}\right) & =F(N, \hat{b}, \hat{\mu})+F\left(N^{\prime}, \hat{b}^{\prime}, \hat{\mu}\right),
\end{aligned}
$$

where $\hat{\mu}=\left(\mu_{j}\right)_{j=1 \ldots m}, \hat{b}=\left(b_{j}\right)_{j=1 \ldots m}$, symbol $\oplus$ denotes the componentwise addition. Indeed, the first equality was proved in Sect. 16, while the second equality follows from Property (P3) of the spectral flow.

Hence $F$ is a bilinear function, and therefore there is a homomorphism from $\mathbb{Z}^{m+1} \otimes$ $M$ to $\mathbb{Z}$ such that $F$ can be represented as the composition

$$
S \times M \hookrightarrow \mathbb{Z}^{m+1} \times M \rightarrow \mathbb{Z}^{m+1} \otimes M \rightarrow \mathbb{Z}
$$

where the first arrow is induced by the natural embedding of $S$ into $\mathbb{Z}^{m+1}$, and the second arrow is the canonical map of the direct product to the tensor product.

Let us consider operator (2) with boundary condition (3). If $\left(\mathbb{D}+Q_{t}\right) u=0$ and $i\left(n_{1}+i n_{2}\right) u^{+}=B u^{-}$on $\partial X$, then

$$
\begin{aligned}
\int_{\partial x}\left\langle B(x) u^{-}, u^{-}\right\rangle d s= & \int_{\partial x}\left\langle i\left(n_{1}+i n_{2}\right) u^{+}, u^{-}\right\rangle d s \\
= & \int_{X}\left\langle\left(-i\left(\partial_{1}+i \partial_{2}\right)+q_{t}\right) u^{+}, u^{-}\right\rangle d x^{1} d x^{2} \\
& -\int_{X}\left\langle u^{+},\left(-i\left(\partial_{1}-i \partial_{2}\right)+\bar{q}_{t}\right) u^{-}\right\rangle d x^{1} d x^{2}=0,
\end{aligned}
$$

where ds is the length element on $\partial X$.

Suppose now that the sign of $B$ is the same on all boundary components. Then from the last equality we have $u^{-} \equiv 0$ on $\partial X, u^{+}=-i\left(n_{1}-i n_{2}\right) B u^{-} \equiv 0$ on $\partial X$. Thus $u \equiv 0$ on $X$ by the weak inner unique continuation property of the Dirac operator [8]. So $\left(\mathbb{D}+\mathrm{Q}_{t}, \mathrm{~B}\right)$ has no zero eigenvalues at any $t$, and by Property (P0) sf $\left(\mathbb{D}+Q_{t}, B\right)=0$. Finally we obtain $F(1, \hat{0}, \hat{\mu})=F(1, \hat{1}, \hat{\mu})=0$ at any $\hat{\mu}$, where we denote $\hat{0}=(0, \ldots, 0), \hat{1}=(1, \ldots, 1) \in \mathbb{Z}^{\mathrm{m}}$.

Let us consider the group $M^{\prime}$ which is the quotient of $\mathbb{Z}^{m+1}$ by the subgroup spanned by elements $(1, \hat{0}),(1, \hat{1}) \in \mathbb{Z}^{m+1}$. Note that $M^{\prime}$ coincides with the quotient group $\mathbb{Z}^{\mathrm{m}} /\langle\hat{1}\rangle$, so it is naturally isomorphic to the Abelian group $\operatorname{Hom}(M, \mathbb{Z})$ of all homomorphisms of $M$ to $\mathbb{Z}$.

By previous arguments, there exists a homomorphism $\widetilde{F}: M^{\prime} \otimes M \rightarrow \mathbb{Z}$ such that $F$ is the composition of the following homomorphisms:

$$
S \times M \hookrightarrow \mathbb{Z}^{m+1} \times M \rightarrow \mathbb{Z}^{m+1} \otimes M \rightarrow M^{\prime} \otimes M \stackrel{\widetilde{F}}{\rightarrow} \mathbb{Z}
$$

where the first two arrows are the same as in (20), and the third arrow is induced by the natural projection $\mathbb{Z}^{\mathrm{m}+1} \rightarrow \mathrm{M}^{\prime}$. 


\section{Invariance under the Action of Symmetric Group}

Let $\operatorname{Diff}^{+}(X)$ be the group of all diffeomorphisms of $X$ preserving orientation, $f \in$ $\operatorname{Diff}^{+}(X)$. As was shown in Sect. $14, F\left(N, f^{*} B, f^{*} \mu\right)=F(N, B, \mu)$, and hence

$$
F\left(N, f^{*} \hat{b}, f^{*} \hat{\mu}\right)=F(N, \hat{b}, \hat{\mu})
$$

where $f^{*}$ acts on $\hat{b}$ and $\hat{\mu}$ by permutation of the coordinates, corresponding to the permutation of the boundary components of $X$ by $f$. It is well known that any permutation of the boundary components of $X$ is realized by some element of $\operatorname{Diff}^{+}(X)$. Thus $F(N, \hat{b}, \hat{\mu})$ is invariant under the action of symmetric group $S_{m}$ (the group of permutations of $m$ elements) on $(\hat{b}, \hat{\mu})$ by permutations of the coordinates.

All permutations of the coordinates leave the element $\hat{1}$ of $\mathbb{Z}^{\mathrm{m}}$ invariant, so $S_{\mathrm{m}}$ acts on $M^{\prime}=\mathbb{Z}^{m} /\langle\hat{1}\rangle$ in exactly the same way, and $\widetilde{F}$ is invariant under the action of $S_{m}$, too.

Extending $\widetilde{\mathrm{F}}$ by linearity from $M^{\prime} \otimes M$ to $\mathrm{V}^{\prime} \otimes \mathrm{V}, \mathrm{V}^{\prime}=\mathrm{M}^{\prime} \otimes \mathbb{C}=\mathbb{C}^{\mathrm{m}} /\langle\hat{1}\rangle$, $\mathrm{V}=\mathrm{M} \otimes \mathbb{C}=\left\{v \in \mathbb{C}^{\mathrm{m}}: \sum v_{\mathrm{j}}=0\right\}$, we obtain a homomorphism $\widetilde{\mathrm{F}}_{\mathbb{C}}: \mathrm{V}^{\prime} \otimes \mathrm{V} \rightarrow \mathbb{C}$, coinciding with $\widetilde{\mathrm{F}}$ on the lattice $M^{\prime} \otimes M \subset \mathrm{V}^{\prime} \otimes \mathrm{V}$. Obviously, $\widetilde{\mathrm{F}}_{\mathbb{C}}$ is invariant with respect to the action of $S_{m}$ on $V^{\prime} \otimes V$ as well.

$V^{\prime}$ and $\operatorname{Hom}_{\mathbb{C}}(V, \mathbb{C})$ coincide as representations of $S_{m}$, so the vector space of all invariant homomorphisms from $V^{\prime} \otimes V$ to $\mathbb{C}$ is isomorphic to the vector space of all equivariant homomorphisms $V \rightarrow V$. But the latter space is 1-dimensional by Schur's lemma, because $V$ is an irreducible representation of $S_{m}[10]$. So $\widetilde{F}_{\mathbb{C}}\left(v^{\prime} \otimes v\right)=c \sum_{j} v_{j}^{\prime} v_{j}$ for some constant $c \in \mathbb{C}$, and $F(N, \hat{b}, \hat{\mu})=c \sum b_{j} \mu_{j}$, where $c$ depends only on $m$.

On the other hand, $F$ is integer-valued and, in particular, $c=F(1,(0,1),(-1,1)) \in \mathbb{Z}$.

Finally, we obtain

$$
\operatorname{sf}\left(D+Q_{t}, B\right)_{t \in[0,1]}=c_{m} \sum_{j=1}^{m} b_{j} \mu_{j},
$$

where $c_{m}$ is an integer constant depending on $m$ only, and Theorem 3 is proved.

Acknowledgements. The author is very grateful to M.I. Katsnelson for attracting the author's attention to this circle of questions and for explaining that these problems are of potential importance for condensed matter physics. The latter served as an important motivation for this work. Conversations with M.I. Katsnelson helped to understand what kind of difficulties a physicist may have while reading a mathematical paper. Hopefully, this has led to a more physicist-friendly style of this paper. M.I. Katsnelson also informed the author about the papers $[1,3]$. The main part of the text was written during the author's visit to Radboud University Nijmegen at the invitation of M.I. Katsnelson and with financial support from the Stichting voor Fundamenteel Onderzoek der Materie (FOM).

The author thanks M. Lesch and F.V. Petrov for their help with subtle questions of operator theory and the theory of Sobolev spaces. The author is very grateful to N.V. Ivanov for his continuous support and his efforts to improve the exposition in this paper. The author is thankful to M. Braverman, A. Gorokhovsky, M.E. Kazaryan, and I.A. Panin for stimulating discussions on various topics related to this paper. The author is also very thankful to I. Marshall for the correcting the English language in the final version of the paper.

This work was partially supported by the RFBR grant 09-01-00139-a (Russia), and by the Program for Basic Research of Mathematical Sciences Branch of Russian Academy of Sciences (project 12-T-1-1003). It was partially done during the author's stay at the Max Planck Institute for Mathematics (Bonn, Germany); the author is grateful to this institution for hospitality and the excellent working conditions. 


\section{References}

1. Akhmerov, A.R., Beenakker, C.W.J.: Boundary conditions for Dirac fermions on a terminated honeycomb lattice. Phys. Rev. B 77(8), 085423 (2008)

2. Atiyah, M.F., Patodi, V.K., Singer, I.M.: Spectral asymmetry and Riemannian geometry. III. Math. Proc. Cambridge Phil. Soc. 79(1), 71-99 (1976)

3. Berry, M.V., Mondragon, R.J.: Neutrino billiards: time-reversal symmetry-breaking without magnetic fields. Proc. Roy. Soc. A 412(1842), 53-74 (1987)

4. Booss-Bavnbek, B., Furutani, K.: The Maslov index: a functional analytical definition and the spectral flow formula. Tokyo J. Math. 21(1), 1-34 (1998)

5. Booss-Bavnbek, B., Lesch, M., Phillips, J.: Spectral flow of paths of self-adjoint Fredholm operators. Nucl. Phys. B Proc. Supp. 104, 177-180 (2002)

6. Booss-Bavnbek, B., Lesch, M., Phillips, J.: Unbounded Fredholm operators and spectral flow. Canadian J. Math. 57(2), 225-250 (2005)

7. Booss-Bavnbek, B., Lesch, M., Zhu, C.: The Calderon projection: new definition and applications. J. Geom. Phys. 59(7), 784-826 (2009)

8. Booss-Bavnbek, B., Wojciechowski, K.P.: Elliptic boundary problems for Dirac operators. Basel-Boston: Birkhauser, 1993

9. Brüning, J., Lesch, M.: On boundary value problems for Dirac type operators: I. Regularity and Self-Adjointness. J. Funct. Anal. 185(1), 1-62 (2001)

10. Fulton, W., Harris, J.: Representation theory. A first course. Graduate Texts in Mathematics, Readings in Mathematics, 129. Berlin-Hedilberg-New York: Springer-Verlag, 1991

11. Katsnelson, M.I., Nazaikinskii, V.E.: The Aharonov-Bohm effect for massless Dirac fermions and the spectral flow of Dirac type operators with classical boundary conditions. Preprint: arXiv:1204.2276v1 [math.AP], 2012; Teoret. Mat. Fiz. 172(3), 437-453 (2012); Eng. trans. in. Theoret. Math. Phys. 172(3), 1263-1277 (2012)

12. Lesch, M.: The uniqueness of the spectral flow on spaces of unbounded self-adjoint Fredholm operators. In: Booss-Bavnbek, B., Grubb, G., Wojciechowski, K.P. (eds.) Spectral Geometry of Manifolds with Boundary and Decomposition of Manifolds, AMS Contemporary Math Proceedings 366, 193-224, (2005), pp. 193-224

13. Nicolaescu, L.: The Maslov index, the spectral flow, and decomposition of manifolds. Duke Math. J. 80(2), 485-533 (1995)

14. Phillips, J.: Self-adjoint Fredholm operators and spectral flow. Canadian Math. Bull 39(4), 460-467 (1996)

15. Prokhorova, M.: The spectral flow for Dirac operators on compact planar domains with local boundary conditions. arXiv:1108.0806v1 [math-ph], 2011

16. Prokhorova, M.: The spectral flow for first order elliptic operators on a compact surface. In preparation

17. Strichartz, R.S.: Multipliers on fractional Sobolev spaces. J. Math. and Mech. 16(9), 1031-1060 (1967)

18. Yoshida, T.: Floer homology and splittings of manifolds. Ann. Math. 134, 277-323 (1991)

Communicated by B. Simon 